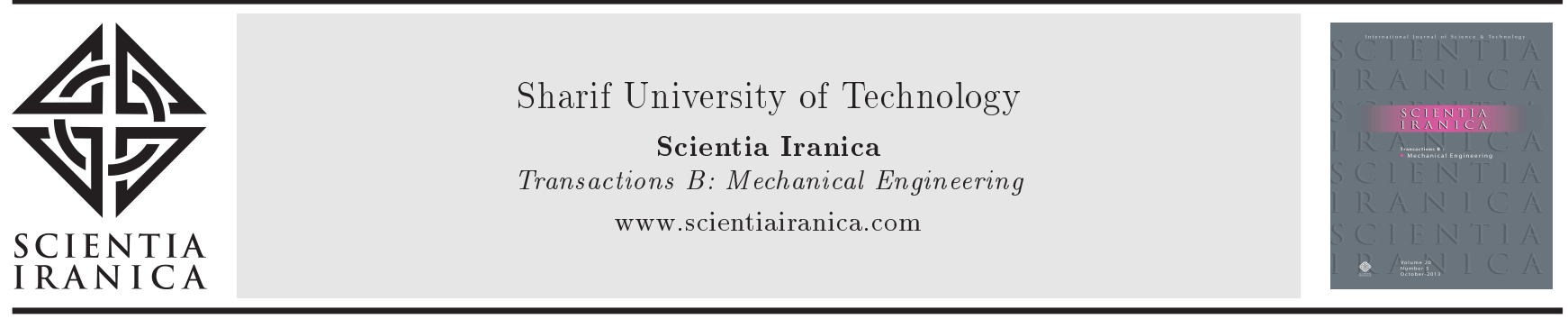

\title{
The effect of ductile damage on plastic behavior of a rotating disk with variable thickness subjected to mechanical loading
}

\author{
R. Akbari Alashti* and S. Jafari \\ Department of Mechanical Engineering, Babol University of Technology, Babol, P.O. Box 484, Iran.
}

Received 17 April 2014; received in revised form 12 February 2015; accepted 13 April 2015

\section{KEYWORDS \\ Rotating disk; \\ Ductile damage; \\ Successive elastic \\ solution; \\ Finite element \\ analysis; \\ Angular velocity.}

\begin{abstract}
In this work, the effect of ductile damage on distributions of strain and displacement components in rotating annular disks with variable thicknesses under plane stress conditions is studied using semi-analytical and finite element methods. The plastic behavior of disks under mechanical loading is studied on the basis of continuum damage mechanics. The semi-analytical method is developed using Prandtl-Reuss relations, the method of successive elastic solution, and the damage plasticity model proposed by Xue and Wierzbicki that is also used for finite element analysis. The proposed damage plasticity model incorporates the effects of both hydrostatic stress and the Lode angle to define the fracture envelope. The results obtained by the semi-analytical method are then compared with the results obtained by the finite element method. Numerical calculations for different ranges of thickness parameter, with and without damage effects, are carried out and the results are compared. It is shown that the damage has a significant effect on values of maximum von Mises stress and the limit angular velocity of annular disks. Results of the present study confirm the credibility of the proposed model in predicting the damage limit angular velocity, and can be extended to other states of loading.
\end{abstract}

(C) 2016 Sharif University of Technology. All rights reserved.

\section{Introduction}

Estimation of stress and strain distributions in disks rotating at high speeds is an important subject, due to their wide applications in engineering components such as gears, turbine rotors, flywheels, etc. Theoretical research into the behavior of such structures has received appreciable focus, and the topic has been considered in many standard and advanced textbooks [1-4], which mostly concentrate on the analytical method and finite element simulations [4-21]. Furthermore, to the knowledge of the authors, no research work has been

\footnotetext{
* Corresponding author. Tel.: +981113234205; Fax: +981113234201

E-mail addresses: raalashti@nit.ac.ir (R. Akbari Alashti); jaafari.sanaz@yahoo.com (S. Jafari)
}

presented on the effects of ductile damage on the plastic behavior and limit angular velocity of a rotating disk.

The theoretical treatment of elastic-plastic rotating disks was started by Laszlo [5] and, since then, interest in this problem has never ceased [521]. Gamer [6] proposed a stable analytical solution for the elastic-plastic response of a rotating solid disk with uniform thickness. In his research, Tresca's yield criterion, plane stress condition, linear strain hardening material behavior and its associate flow rule were assumed. These works were extended to studies on annular and solid disks with variable thickness and density $[7,8]$. The fully plastic state of a solid disk with variable thickness was investigated by Guven [9].

Analytical solutions for disks with a convex form of thickness variation obeying exponential and power functions were presented by Orcan and Eraslan [10]. 
They also investigated the elastic-plastic deformation of solid disks with variable thickness in concave form [11]. In these works, it was shown that the deformation behavior of a solid disk with varying thickness is different from that of a disk with constant thickness, in such a way that three different stages of elasticplastic deformation occur. The numerical results of the complete plastic state of a solid disk with exponentially varying thickness have been obtained using von Mises and Tresca criteria [12]. Eraslan carried out numerical studies on the mechanical behavior of annular disks with various thickness profiles, including hyperbolic, exponential and power forms [13]. In another work, he studied the elastic-plastic deformation of rotating annular disks of variable thickness with free, pressurized and radially constrained boundary conditions [14].

Hojjati and Jafari studied the variational iteration solution of the elastic behavior of rotating disks with non-uniform thickness and density [15]. Hojjati and Hassani employed the Variable Material Property method (VMP) on analytical and numerical analyses of rotating disks with variable thickness and density made of elastic-linear hardening material [16]. Adomian's Decomposition (ADM) and Homotopy Perturbation Methods (HPM) have also been used by Hojjati and Jafari for the solution of elastic [17] and elastic- linear strain hardening [18] states of rotating annular disks with variable thickness and density. Hojjati et al. considered functionally graded rotating disks subjected to thermo-mechanical loadings by Variable Material Properties (VMP), Runge-Kutta's (RK) and Finite Element (FE) methods [19]. Ekhteraei Toussi et al. [20] worked on the elastic-plastic deformation analysis of a rotating disk beyond its limit speed and demonstrated the difference between the material models in the prediction of rotating disk plastic failure. Jafari et al. [21] presented classical and modern optimization methods in minimum weight design of an elastic rotating disk with variable thickness and density. They used Karush-Kuhn-Tucker, Simulated Annealing (SA) and Particle Swarm Methods (PSO) and found that the performance of PSO and SA methods are simpler and supply more flexibility.

Semi-empirical methods are developed by Robinson [22] in order to easily find the burst speed by knowing the ultimate tensile strength and the mean hoop stress. According to Robinson, a criteria burst occurs when the mean hoop stress on a disk section becomes equal to the nominal tensile strength of the material, determined from uniaxial tensile stress [22]. Recently, large deformation analysis of rotating disks by Abaqus software [23], and theoretical analysis of the elastic-plastic interface of rotating disks [24] was undertaken by $\mathrm{Hu}$. In his research, two types of differential material disk were analyzed and the formula for the large deformation analysis of a rotating disk was derived. Finally, the bursting speeds of a rotating disk were given out.

From experimental observations, it was found that damage resulting from the plastic deformation in a ductile metal is mainly due to the formation of microvoids [25]. Furthermore, accumulation of damage, initiation of fracture as a result of the accumulation of ductile plastic damage, and crack propagation, are the three phases of the ductile failure of structures $[25,26]$. Ductile fracture refers to fractures where materials experience large plastic deformation and exhibit high ductility in the concerned region where structural damage occurs [25,26-32]. For small and moderate plasticity, the damage effect on the matrix strength curve is negligible, and the material strength curve may be considered equal to that proposed by classic continuum mechanics. However, in the case of large plastic deformations, the damage effect must be considered in the prediction of material deterioration [25,26-32]. Therefore, in finite element simulations of disks under mechanical loading, it is essential to use the modified stress-strain curve.

Continuous efforts have been made by researchers, using various constitutive models, towards the finite element simulation of ductile fracture problems. The micromechanical model that relies on the global mechanical response of some kind of microscopic representative volume structure, such as models proposed by Gurson [33] and Tvergaard [34], is one type of such damage model. Another type of damage model, based on cumulative strain damage, was used by Johnson and Cook [35] and Wilkins et al. [36]. The Continuum Damage Mechanics (CDM) model that includes ductile damage under the yield condition to reflect material deterioration, as undertaken by Lemaitre [26], is a later extension of this line of damage model.

The Finite Element Method Of Lines (FEMOL) in fracture mechanics is presented in $\mathrm{Hu}$ and Moran [37], in detail, in a set of examples such as edgecrack plate, central-crack plate, and plate with cracks emanating from a hole under tensile or under combination loads of tensile and bending. They have shown that FEMOL is a useful method for studying fracture mechanics problems [37]. Moreover, the Element-FreeGalerkin (EFG) method for problems in 3D fracture is presented by Sukumar et al. [38]. Fracture initiation by ductile failure mechanisms in plates made of 4340 steel is studied by Narasimhan et al. [39] with a 3D numerical method.

Experimental results have shown that four parameters are quite important in prediction of fracture initiation by ductile fracture models. These are, namely, the damage rule, the softening effect, the Lode angle (the third deviatoric stress invariant) parameter, and the hydrostatic pressure dependence function [25,2732 . It is also observed that the fracture strain is 
loading path dependent, hence, the damage rule is employed to quantify the amount of damage accumulated along the corresponding loading path [25,27-32]. The softening effect becomes one of the most important parameters when a ductile material is subjected to large plastic deformation. As the plastic deformation increases, micro cracks are initiated and propagated. Hence, the softening effect must be considered using an appropriate weakening function in the ductile fracture model [25,27-32]. Hydrostatic pressure and Lode angle parameter have significant effects on the shape of the fracture envelope in the principal stress space. The Lode angle parameter modifies the cross section of the fracture envelope on the octahedral plane, based on the current deviatoric stress state of the material. The hydrostatic pressure parameter is important, since materials exhibit higher values of ductility when subjected to compressive hydrostatic pressure [25]. For this reason, at each step of the loading process, the shape of the fracture envelope is improved by the hydrostatic pressure parameter.

One of the recent damage plasticity models in the CDM category, which includes these four effects, is the one proposed by Xue and Wierzbicki (X-W) [25,27-32]. Xue et al. established the well-known damage plasticity model that shows the significance of the ductile damage effect in the performance of rotating disks. If we intend to design a rotating disk for practical purposes, we must consider an appropriate damage model to obtain a reasonably correct estimation of the limit load (i.e. the limit angular velocity). In this study, it is assumed that the maximum limit angular velocity of the disk occurs at the incipient of the disk fracture.

In analysis of the elastic-plastic response of an annular disk, the von Mises yield criterion and its associated flow rule are used with the assumption of linear hardening material behavior. In this paper, a damage plasticity model proposed by Xue and Wierzbicki is employed for semi-analytical and finite element investigation of the plastic strain level and limit angular velocity of a rotating disk subjected to mechanical loading. In the semi-analytical method, the PrandtlReuss relations and the method of successive elastic solution are used to model the plastic deformation of the rotating disk, and the Finite Difference Method (FDM) is introduced to solve the governing equations. The proposed damage plasticity model incorporates pressure sensitivity and Lode angle dependence and falls into the category of CDM theory. Deterioration of material in the CDM theory is explained by an internal variable known as damage. Plastic deformation that contributes to damage is computed by integrating the damage rate obtained at the current loading. To determine the nonlinearity in damage accumulation, a power law damage rule is assumed. The damage related weakening factor is considered to describe material deterioration. The material and damage parameters for Aluminum 2024-T351 alloy are obtained from standard experimental tests [25]. In this study, the disk is assumed to have a varying thickness profile. The disk is assumed to be radially constrained at the inner surface and free of any traction at the outer surface. The results obtained by the semi-analytical method, with and without the damage effect, are obtained and compared with the finite element results.

\section{Theoretical background}

\subsection{Governing equations of a rotating disk}

In this research, the behavior of a variable thickness rotating disk under isothermal conditions is studied, i.e. no thermal stresses exist, and only stresses caused by the rotation are considered. The cylindrical coordinate system $(r, \theta, z)$ is considered for the rotating disk. In the steady state condition, and assuming that the stresses do not vary over the thickness of the disk, the force equilibrium in the radial direction results in the following equation $[2,16-17]$ :

$$
\frac{d}{d r}\left(h(r) r \sigma_{r}\right)-h(r) \sigma_{\theta}=-h(r) \rho \omega^{2} r^{2}
$$

where $\omega, \rho=\rho_{0}$, and $h(r)$ are angular velocity, density and thickness of the disk, respectively. With the assumption of plane stress and small deflection condition, the strain-displacement relation is defined as [2]:

$$
\varepsilon_{r}=\frac{d u}{d r}, \quad \varepsilon_{\theta}=\frac{u}{r} .
$$

In this study, an elastic-linear hardening [2] model is used to model the stress-strain curve of the disk material, as shown in Figure 1:

$$
\begin{cases}\varepsilon=\frac{\sigma}{E} & \sigma\left\langle\sigma_{\circ}\right. \\ \varepsilon=\frac{\sigma_{\circ}}{E}+\frac{1}{E_{t}}\left(\sigma-\sigma_{\circ}\right) & \sigma\rangle \sigma_{\circ}\end{cases}
$$

where $\sigma_{\circ}$ and $E_{t}$ are the yield strength of the material

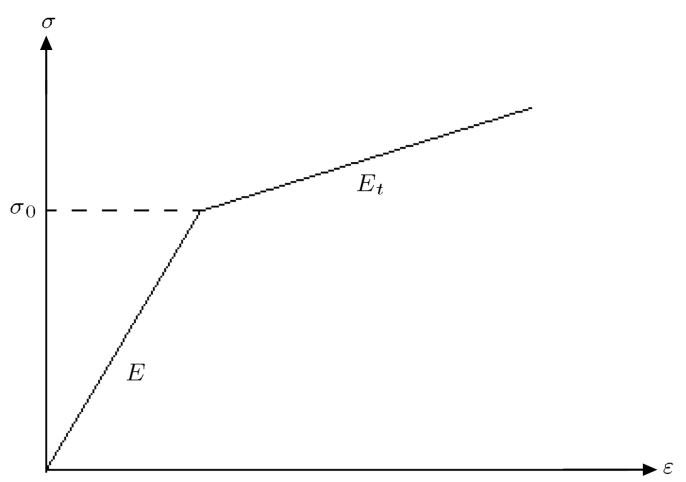

Figure 1. Idealized stress-strain curve for a linear hardening material. 
and tangent modulus, respectively. By using Eq. (3), the following relation for the equivalent stress, $\sigma_{e}$, that occurs in the plastic region of the disk can be easily obtained:

$$
\sigma_{e}=\sigma_{\circ}\left(1+\eta \varepsilon_{e q}^{p}\right),
$$

where $\varepsilon_{e q}^{p}$ is the equivalent plastic strain and $\eta=$ $E E_{t} /\left[\sigma_{0}\left(E-E_{t}\right)\right]$ indicates the hardening parameter [14-18,40].

The disk is supposed to be symmetric, with respect to the mid plane, and its thickness profile to vary as a function of the radius $(r)[6,16,17]$ :

$$
h(r)=h_{\circ}(r / b)^{-n},
$$

where $n$ is the geometric parameter $(0 \leq n \leq 1), h_{\circ}$ is the thickness of the disk at $r=b$, and $b$ is the outer radius of the disk. It is obvious that a uniform thickness disk is simply obtained by setting $n=0$. Figure 2 shows the geometry of a rotating disk with variable thickness for different values of geometric parameter, $n$. The geometry and material properties of the disk under study in this research are shown in Table 1.

\subsection{Damage plasticity model}

2.2.1. Formulation of $X-W$ damage plasticity model The results of experiments carried out on the behavior of ductile materials reported in the literature show that for better prediction of material behavior along the

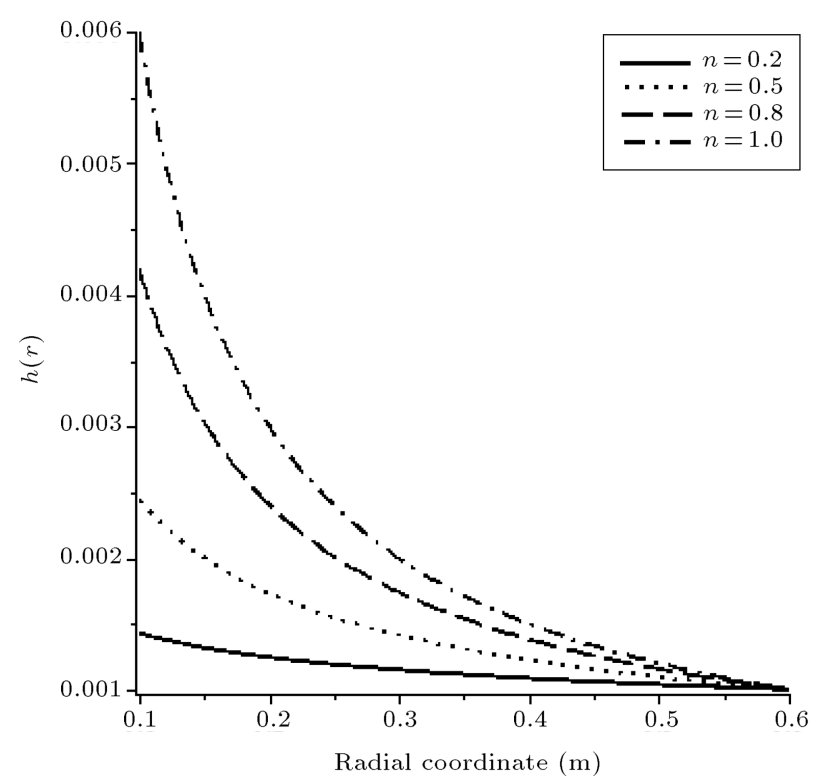

Figure 2. Disk profile for different geometric parameter $n$. loading path, an appropriate damage model must be included in the classical plasticity theory [18-30]. The main object of this study is to investigate the effects of pressure and Lode angle parameter in the plasticity model, with damage rule and softening effects that were not accounted previously. The damage is assumed to be isotropic and quantified by the scalar parameter, $D$. In the principal stress space, the hydrostatic pressure $(p)$, von Mises equivalent stress $\left(\sigma_{e q}\right)$ and Lode angle parameter $\left(\theta_{L}\right)$ are defined in terms of $\sigma_{1}, \sigma_{2}$ and $\sigma_{3}$, i.e. the maximum, intermediate and minimum principal stresses, and $s_{1}, s_{2}$ and $s_{3}$, i.e. the maximum, intermediate and minimum principal deviatoric stresses, as follows [25,27-32]:

$$
\begin{aligned}
& p=-\frac{1}{3}\left(\sigma_{1}+\sigma_{2}+\sigma_{3}\right), \\
& \sigma_{e q}=\frac{1}{\sqrt{2}} \sqrt{\left(\sigma_{1}-\sigma_{2}\right)^{2}+\left(\sigma_{1}-\sigma_{3}\right)^{2}+\left(\sigma_{2}-\sigma_{3}\right)^{2}}, \\
& \theta_{L}=\tan ^{-1}\left\{\frac{1}{\sqrt{3}}\left(2 \frac{S_{2}-S_{3}}{S_{1}-S_{3}}\right)-1\right\} .
\end{aligned}
$$

\subsubsection{Damage rule}

Using the ductile damage parameter, $D$, one can define the fracture criterion by setting the inequality, $D \geq$ $D_{c r}$, where $D_{c r}$ is the material constant. The fracture criterion is generally expressed in the non-dimensional form of $\left(D / D_{c r}\right) \geq 1$. The damage is not necessarily linear, with respect to the equivalent plastic strain, and, hence, the power law function of damage potential of the following form may be considered [27-32]:

$$
D=\left(\varepsilon_{e q}^{p} / \varepsilon_{f}\right)^{m} .
$$

For a known, $\varepsilon_{f}$, the incremental damage evolution law can be derived:

$$
d D=m\left(\frac{\varepsilon_{e q}^{p}}{\varepsilon_{f}}\right)^{m-1} \frac{d \varepsilon_{e q}^{p}}{\varepsilon_{f}} .
$$

For an arbitrary plastic loading path, the damage is obtained by the following integral:

$$
D=\int_{0}^{\varepsilon_{c}} d D \leq 1
$$

where $m$ is the material parameter, $\varepsilon_{f}$ is the fracture strain envelope defined on the stress state in the next

Table 1. Geometry and material properties of the rotating disk.

\begin{tabular}{ccccccccc}
\hline $\begin{array}{c}\boldsymbol{a} \\
(\mathbf{m})\end{array}$ & $\begin{array}{c}\boldsymbol{b} \\
(\mathbf{m})\end{array}$ & $\begin{array}{c}\boldsymbol{\rho}_{\circ} \\
\left(\mathrm{kg} / \mathbf{m}^{\mathbf{3}}\right)\end{array}$ & $\boldsymbol{h}(\boldsymbol{r})$ & $\boldsymbol{\rho}(\boldsymbol{r})$ & $\begin{array}{c}\boldsymbol{E} \\
(\mathbf{G P a})\end{array}$ & $\begin{array}{c}\boldsymbol{E}_{\boldsymbol{t}} \\
(\mathbf{G P a})\end{array}$ & $\boldsymbol{v}$ & $\begin{array}{c}\boldsymbol{\sigma}_{\circ} \\
(\mathbf{M P a})\end{array}$ \\
\hline 0.1 & 0.6 & 2800 & $h_{\circ}(r / b)^{-n}$ & $\rho_{\circ}$ & 70 & 35 & 0.3 & 300 \\
\hline
\end{tabular}


sections, $\varepsilon_{c}$ is the critical strain at which fracture occurs, and $\varepsilon_{e q}^{p}$ is the accumulated equivalent plastic strain. It is assumed that $D=0$ for the intact material and $D=1$ for the complete loss of load carrying capacity, i.e. fracture occurrence [27-32].

\subsubsection{Material deterioration}

When the damage occurs, due to the reduction of the effective load carrying area, the material strength is decreased $[25,26]$. This effect can be modeled with the material weakening due to plastic damage that is incorporated as a scalar function to the matrix strength. On the basis of CDM theory, the constitutive equation of the damaged material can be derived from the modified yield potential function:

$$
\varphi=\sigma_{e q}-w(D) \sigma_{M}
$$

We include a particular form of weakening effect by the weakening factor of $\left(1-D^{\beta}\right)$, i.e.:

$$
\sigma_{e q}=\left(1-D^{\beta}\right) \sigma_{M}
$$

where $\sigma_{M}$ is the matrix strength and $\beta$ is the weakening factor that is assumed to be a non-negative constant [25,27-32].

\subsubsection{Cylindrical decomposition of damage}

In the cylindrical decomposition method, pressure, Lode angle, and plastic strain and its rate are used to determine the damage rate. The pressure sensitivity and Lode angle dependence of the fracture strains are included by a pressure dependence function, $\mu_{p}(p)$, and a Lode angle dependence function, $\mu_{\theta}(\theta)$, respectively. The pressure and the Lode angle are orthogonal in the principal stress space. It is supposed that pressure sensitivity and Lode angle dependency on the fracture strain are independent of each other. The fracture envelope obtained by multiplication of pressure sensitivity and the Lode dependency function is assumed to take the following form:

$$
\varepsilon_{f}=\varepsilon_{f 0} \mu_{p}(p) \mu_{\theta}(\theta),
$$

where $\varepsilon_{f 0}$ is the reference fracture strain demonstrated by zero mean stress tension.

The ratio of the mean stress to the equivalent von Mises stress is defined as the stress triaxiality, i.e. $\left(\sigma_{m} / \sigma_{e q}\right)$. Materials exhibit higher values of ductility when subjected to compressive hydrostatic pressure. The damage resulting from plastic deformation in a ductile material is mainly due to the growth of microvoids. At high stress triaxiality, the void growth is considerably higher than in the case of low stress triaxiality [25,27-32], hence, the pressure effect on the fracture strain is sometimes explained by the stress triaxiality ratio. The pressure dependence function for a $(\mathrm{X}-\mathrm{W})$ damage plasticity model is the result of
Bridgman uniaxial tension under pressure tests. In the present study, a logarithmic form of the pressure dependence function is defined as follows:

$\mu_{p}(p)$

$$
= \begin{cases}\left(1-q \operatorname{Ln}\left(1-p / p_{\text {Lim }}\right)\right), & p \geq p_{\lim }[1-\exp (1 / q)] \\ 0 & p<p_{\lim }[1-\exp (1 / q)]\end{cases}
$$

The first kind of Lode angle dependence function is:

$$
\mu_{\theta}(\theta)= \begin{cases}\frac{\sqrt{\mathcal{X}^{2}-\mathcal{X}+1}}{1+(\sqrt{3} / \gamma-2) \mathcal{X}} & 0 \leq \mathcal{X} \leq 0.5 \\ \frac{\sqrt{\mathcal{X}^{2}-\mathcal{X}+1}}{1+(\sqrt{3} / \gamma-2)(1-\mathcal{X})} & 0.5 \leq \mathcal{X} \leq 1\end{cases}
$$

in which $\mathcal{X}$ is the relative ratio of the principal deviatoric stresses defined as:

$$
\mathcal{X}=\frac{S_{2}-S_{3}}{S_{1}-S_{3}}
$$

where $q$ and $p_{\text {Lim }}$ are material constants, $p$ is the current hydrostatic pressure in Eq.(15), and $\gamma$ is material constant in Eq.(16) [27-32].

The fracture envelope in the space of plastic strain and the mean stress is demonstrated in Figure 3, in which the vertical and horizontal axes represent components of the mean stress and the principal plastic strain, respectively, the thick solid line shows the pressure dependence of the material, and the thick dash

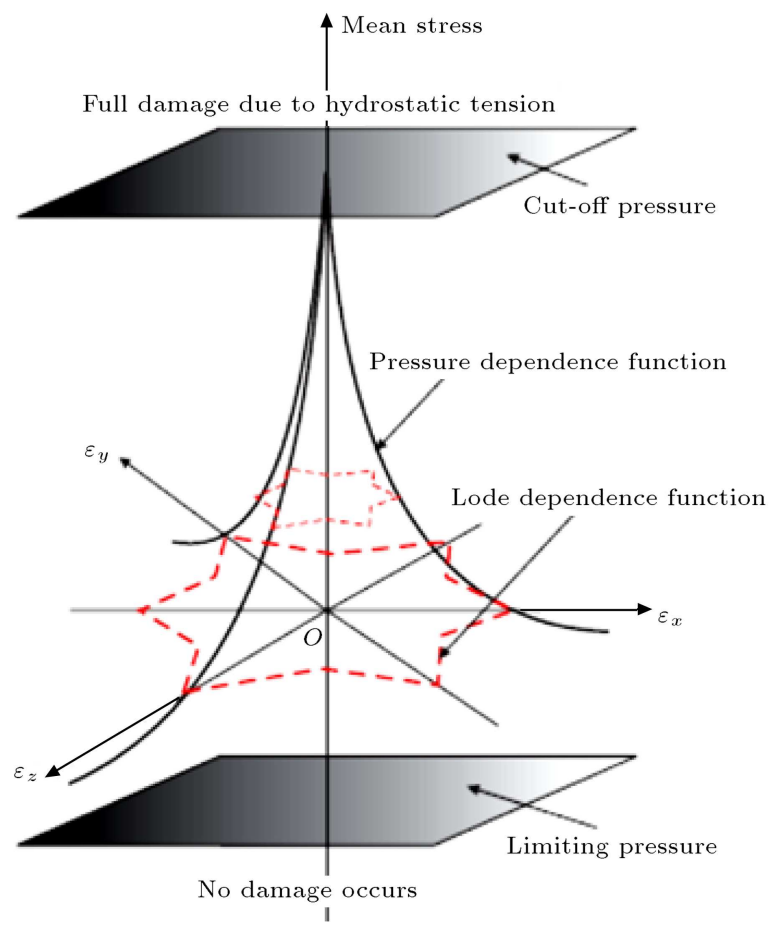

Figure 3. Three dimensional damage strain envelope, mean stress - plastic strain space. 
line depicts the Lode angle dependence. The failure strain is a decaying function with respect to increasing mean stress. It could be shown from Eq. (15) that the failure strain approaches to zero when the hydrostatic pressure reaches the cut-off pressure:

$$
p_{\text {cut-off }}=p_{\text {lim }}\left(1-\exp ^{1 / q}\right),
$$

where $p_{\lim }$ is the limiting pressure beyond which the material will not fail under the uniaxial tensile condition, and $p_{\text {cut-off }}$ is the cut-off pressure above which fracture occurs in hydrostatic tension. As shown in Figure 3, the fracture locus is placed between the limiting and cut-off pressures. On an octahedral plane of cut-off pressure, the fracture locus shrinks to a single point at the triad axis.

Each of the ductile damage models have several constant parameters known as damage parameters. These parameters are considered as constants for a given material and are obtained from four standard laboratory tests. The material chosen for this investigation is Aluminum 2024-T351 alloy. Damage parameters for Aluminum 2024-T351 alloy are obtained from standard experimental tests from the research of Xue [25]. For the proposed set of constitutive damage plasticity equations, six material parameters are used in total, namely, the reference strain, $\varepsilon_{f 0}$, parameters for the pressure dependence function, $q, p_{\text {Lim }}$, the parameter for the Lode angle dependence function, $\gamma$, the damage accumulation exponent, $m$, and the parameter for the weakening effect, $\beta$. Xue and Wierzbicki presented a series of un-notched and notched round bars under axial tension, cylinders under axial compression and doubly-grooved plates (transversely plane strain) under uniaxial tension tests, which cover a wide range of mean stresses and Lode angles to calibrate these parameters for Aluminum 2024-T351 alloy [25,31]. A material parameter calibration process is then followed. Parallel numerical studies by finite element simulations for these tests are conducted to evaluate the obtained material parameter from experiments, and to improve the estimated parameters using an iterative "trial-anderror" method [25,31]. Damage characteristics for this material are shown in Table 2.

\subsection{Finite Difference Method (FDM)}

The finite difference method is an effective method for numerical solution of differential equations. The derivatives appearing in the differential equation and boundary conditions are replaced by suitable finite difference approximations. The accuracy of the solution depends on the chosen number of grid points.

Table 2. Damage characteristics of Aluminum 2024-T351 alloy.

\begin{tabular}{ccccccc}
\hline $\boldsymbol{\varepsilon}_{\boldsymbol{f} \mathbf{0}}$ & $\boldsymbol{P}_{\text {Lim }}$ & $\boldsymbol{q}$ & $\boldsymbol{\gamma}$ & $\boldsymbol{m}$ & $\boldsymbol{\beta}$ & Reference \\
\hline 0.8 & $800 \mathrm{MPa}$ & 1.5 & 0.4 & 2 & 2 & Xue $[20]$ \\
\hline
\end{tabular}

One can increase the accuracy of the solution to some desired degree by increasing the number of grid points. However, it involves increasingly tedious mathematical analysis. The basic idea of difference approximations is a Taylor series expansion. To solve differential equations with specified boundary conditions, the interval $[0,1]$ is divided into $N-1$ equal parts [41]. The first derivative of a function, $f(u)$, with respect to $r$, in central difference form, can be written as:

$$
\left.\frac{d u}{d r}\right|_{r=r_{i}}=\frac{u_{i+1}-u_{i-1}}{2 \Delta r}+O\left(h^{2}\right)
$$

While the second derivative of a function, $f(u)$, with respect to $r$, is:

$$
\left.\frac{d^{2} u}{d r^{2}}\right|_{r=r_{i}}=\frac{u_{i+1}-2 u_{i}+u_{i-1}}{\Delta r^{2}}+O\left(h^{2}\right) .
$$

Other finite difference forms of derivatives for function $f(u)$ are available in $[41,42]$. For each grid point in the interval $[0,1]$, the differential equation is written in the finite difference form. Also, the boundary conditions are written in this scheme. Finally, the set of linear algebraic equations can be obtained in the following matrix expression that must be solved using an appropriate method [41]:

$$
A . U=B \text {. }
$$

\section{Finite Element Method (FEM)}

The elastic-plastic analysis of a rotating disk with variable thickness is carried out by the finite element method using a nonlinear approach with quadratic isoparametric elements in the Ansys suite of program [43]. The nonlinear approach used in the finite element simulation consists of both the material and the geometrical nonlinearities. The material is assumed to have a bilinear strain hardening behavior that is defined in the Ansys suite of program, according to the stress-strain data curve shown in Figure 1, using the TB, BISO command. When the damage effect is considered, the material behavior of the disk becomes nonlinear. At each loading step of the loading path, the material property is changed proportional to the corresponding damage variable of the previous loading step, as defined in Eq. (13). Regarding geometric nonlinearities, it is to be noted that the geometric properties of the disk are linear when the thickness of the disk along the radius is constant, i.e. $n=0$. If the thickness of the disk varies along the radius, according to the geometric parameter, $n$, the geometrical model of the disk becomes nonlinear.

The loading is considered to be due to the inertia force caused by rotation. Due to symmetry in the geometry and loading of the disk, it is desirable to 


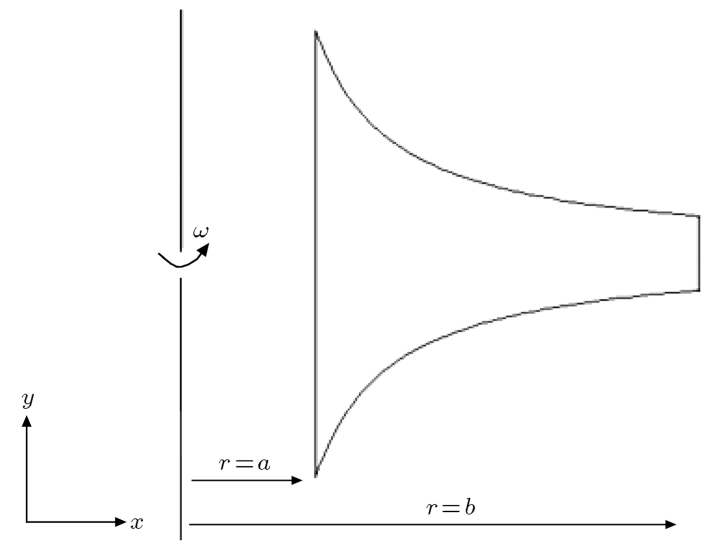

Figure 4. Axisymmetric model of a variable thickness rotating disk.

model just a sector of the disk in order to reduce both the model size and the solution time. Hence, a two dimensional axisymmetric finite element model of the variable thickness rotating disk is created, as shown in Figure 4. The axisymmetric slice is offset in the $x$-direction to simulate a hole in the disk. Radially constrained-free boundary conditions are applied at the inner surface and the outer surface of the disk, respectively.

The finite element model of the disk presented here consists of two-dimensional axisymmetric solid elements, PLANE42, to model a cross-section of the disk that implicitly simulates the behavior of its threedimensional axisymmetric structure. Also, a convergence test is carried out to obtain the accurate number of elements in the variable disk.

\subsection{Implementation of damage evolution in FEM}

Numerical solution of governing equations for the damage plasticity material model requires time discretization. In the present study, an explicit integration procedure is used to convert the rate form of equations to an incremental form. The numerical integration scheme in FE simulations for the (X-W) model is shown by multiple steps in this section. The value of the ductile damage variable is calculated for each element and deformation loading step. This model obeys a damage rule and, by considering the softening effect in the fracture model, the damage variable is included in the plasticity flow for each loading step. Plastic deformation that contributes to damage is calculated by integrating the damage rate measured at the current loading step.

The state of the nonlinear local problem with variables, $\varepsilon_{e q, n}^{p}, D_{n}$ and the stress state, $\sigma_{n}$, are assumed to be known at time, $t_{n}$, for all fracture models. An increment in the strain, $\Delta \varepsilon$, is applied in the time interval. The task is to solve the stress tensor and internal variables at time $t_{n+1}=t_{n}+\Delta t$.
Given: $\left\{\varepsilon_{e q, n}^{p}, D_{n}, \sigma_{n}\right\}$ and $\Delta \varepsilon=\varepsilon_{n+1}-\varepsilon_{n}$ at time $t_{n}$. Calculate: $\left\{\varepsilon_{e q, n+1}^{p}, D_{n+1}, \sigma_{n+1}\right\}$ at time $t_{n+1}=t_{n}+$ $\Delta t$.

Step 1: Material properties definition: Initially, and before loading, for each element in the meshing of the disk, the material property is defined (the number of material properties is equal to the number of elements in the meshing of the disk);

Step 2: Elastic predictor and plastic consistency check: For FE simulation of the ductile fracture model under study, a small value of angular velocity is the first loading step, so that elastic deformation occurs in the disk and, hence, no damage is included. The equivalent von Mises stress of each element for the present loading step is calculated as the trial stress, $\sigma_{n+1}^{\text {trial }}$. The value of $\sigma_{n+1}^{\text {trial }}$ is substituted into the yield function, $\varphi$. If:

$$
\varphi\left(\sigma_{n+1}^{\text {trial }}, D_{n}, \sigma_{M}\left(\varepsilon_{e q, n}^{p}\right)\right) \leq 0
$$

the deformation at this stage of loading is still in the elastic range with no damage, hence:

$$
\left.\begin{array}{c}
\sigma_{n+1}=\sigma_{n+1}^{\text {trial }} \\
\varepsilon_{e q, n+1}^{p}=\varepsilon_{e q, n}^{p}=0 \\
D_{n+1}=D_{n}=0
\end{array}\right\} .
$$

If $\varphi>0$, the deformation is in the elastic-plastic state. For these elements, a plastic correction step is used to enforce the consistency condition and equivalent stress corrections;

Step 3: Return mapping and update the variables: For each element undergoing elastic-plastic deformation, the incremental equivalent plastic strain, $\Delta \varepsilon_{e q}^{p}$, is first calculated. Two internal variables, $\varepsilon_{e q}^{p}$ and $D$, are to be updated for the next loading step.

In the $(\mathrm{X}-\mathrm{W})$ model, the softening effect and the subsequent weakening factor is considered in the $\mathrm{FE}$ simulation. For each loading step, the damage variable and accumulated equivalent plastic strain and stress are updated. The damage at time $t_{n+1}$ is found based on the damage rule, as defined in Eq. (8):

$$
D_{n+1}=D_{n}+m\left(\frac{\varepsilon_{e q, n}^{p}}{\varepsilon_{f, n}}\right)^{m-1} \frac{\Delta \varepsilon_{e q}^{p}}{\varepsilon_{f, n}}
$$

where:

$$
\varepsilon_{f, n}=\varepsilon_{f 0}\left\{\begin{array}{l}
{\left[1-q \operatorname{Ln}\left(1-\sigma_{h_{n}} / p_{\text {Lim }}\right)\right] \frac{\sqrt{\mathcal{X}_{n}^{2}-\mathcal{X}_{n}+1}}{1+\left(\frac{\sqrt{3}}{\gamma}-2\right) \mathcal{X}_{n}}} \\
0 \leq \mathcal{X}_{n} \leq 0.5 \\
{\left[1-q \operatorname{Ln}\left(1-\sigma_{h_{n}} / p_{\text {Lim }}\right)\right] \frac{\sqrt{\mathcal{X}_{n}^{2}-\mathcal{X}_{n}+1}}{1+\left(\frac{\sqrt{3}}{\gamma}-2\right)\left(1-\mathcal{X}_{n}\right)}} \\
0.5 \leq \mathcal{X}_{n} \leq 1
\end{array}\right.
$$


The update on the equivalent plastic strain is:

$$
\varepsilon_{e q, n+1}^{p}=\varepsilon_{e q, n}^{p}+\Delta \varepsilon_{e q}^{p} .
$$

The stress update at time $t_{n+1}$ is calculated based on the updated weakening factor:

$$
\sigma_{n+1}=w\left(D_{n+1}\right) \sigma_{n}=\left(1-D_{n+1}\right)^{\beta} \sigma_{n} .
$$

All necessary field variables $\left\{\sigma_{n+1}, \varepsilon_{e q, n+1}^{p}, D_{n+1}\right\}$ are updated at time $t_{n+1}=t_{n}+\Delta t$.

Step 4: Termination criterion: For the proposed fracture model, the new incremental strain is imposed and Step 1 of the algorithm is restarted until the fracture criterion is satisfied for a given element, i.e. the integral over $D$ reaches the value of one. At this time, the fracture occurs and the calculation is terminated. Angular velocity relating to this situation is considered the critical value for the rotating disk.

\section{Semi-analytical method}

\subsection{Elastic solution}

For the plane stress condition (i.e. $\sigma_{z}=0$ ), the elastic stress-displacement relations are found to be $[2,16]$ :

$$
\begin{aligned}
\sigma_{r} & =\frac{E}{1-v^{2}}\left[\frac{v u}{r}+\frac{d u(r)}{d r}\right], \\
\sigma_{\theta} & =\frac{E}{1-v^{2}}\left[\frac{u}{r}+v \frac{d u(r)}{d r}\right],
\end{aligned}
$$

where $u$ is the radial displacement, $E$ is the modulus of elasticity, $\sigma_{r}$ and $\sigma_{\theta}$ are radial and circumferential components of the stress field, respectively, and $v$ is the Poisson's ratio. Substitution of Eqs. (5), (26), and (27) into Eq. (1) yields the following governing differential equation for the radial displacement:

$$
\begin{aligned}
\frac{d^{2}}{d r^{2}} u(r) & +\frac{(-n+1)}{r} \frac{d}{d r} u(r)-\frac{(n v+1)}{r^{2}} u(r) \\
& =\frac{-\rho_{\circ} \omega^{2} r\left(1-v^{2}\right)}{E} .
\end{aligned}
$$

The above differential equation is valid for elastic deformations of the variable thickness rotating disk. To solve the differential equation by the finite difference method, derivatives of the displacement component in the radial direction are replaced by Eqs. (19) and (20). Finally, the finite difference form of the differential equation is expressed as follows:

$$
\begin{gathered}
\frac{u_{i+1}-2 u_{i}+u_{i-1}}{\Delta r^{2}}+\frac{(-n+1)}{2 r_{i}} \frac{u_{i+1}-u_{i-1}}{\Delta r} \\
-\frac{(n v+1)}{r_{i}^{2}} u_{i}=-\frac{\rho_{0} \omega_{i}^{2} r_{i}\left(1-v^{2}\right)}{E} .
\end{gathered}
$$

The radial direction of the disk $(r \in[a, b])$ is divided into $N$ grid points $(i=[1, N])$. For internal grid points $(i=[2, N-1])$, the FD form of the differential equation is written. In addition, the boundary condition of the rotating disk at the inner and outer surfaces of the disk must be expressed in FD form. The inner surface of the disk is assumed to be attached to a rigid shaft and, hence, the radial displacement at this surface is assumed to be zero. The outer surface of the disk is free of any traction and the radial stress is zero. These boundary conditions are shown as follows:

$$
\begin{aligned}
& i=1, \quad u(a)=0 \rightarrow u_{1}=0, \\
& i=N, \quad \sigma_{r}(b)=0, \quad \frac{u_{N}-u_{N-1}}{\Delta r}+\frac{v u_{N}}{b}=0 .
\end{aligned}
$$

Finally, a system of linear algebraic equations is obtained. By solving the system of equations, the radial displacement of the rotating disk in the radial direction for each grid point is calculated. Now, with strain-displacement and stress-strain relations, one can achieve values of stresses and strains in the rotating disk under elastic limit angular velocity.

\subsection{Plastic solution}

If the angular velocity of the rotating disk is increased beyond the critical value, some part of the disk will deform plastically. For the plastic deformation, relations between stresses and plastic strains can be determined according to the deformation theory in plasticity. The equilibrium equation of the rotating disk for plastic deformation is similar to the elastic state, as in Eq. (1). The stress-strain relations for the plastic deformation are:

$$
\begin{aligned}
& \varepsilon_{r}=\frac{1}{E}\left[\sigma_{r}-v \sigma_{\theta}\right]+\varepsilon_{r}^{p}, \\
& \varepsilon_{\theta}=\frac{1}{E}\left[\sigma_{\theta}-v \sigma_{r}\right]+\varepsilon_{\theta}^{p},
\end{aligned}
$$

where $\varepsilon_{r}$ and $\varepsilon_{\theta}$ are total strains and $\varepsilon_{r}^{p}$ and $\varepsilon_{\theta}^{p}$ are total plastic strains in radial and circumferential directions, respectively. To achieve the Navier form of equilibrium equation, stress components from Eqs. (32) and (33) are substituted into the equilibrium equation, i.e. Eq. (1). Then, total strains are replaced with Eq. (2), and, finally, the Navier form of the equilibrium equation of the rotating disk with plastic deformation is obtained as follows:

$$
\begin{gathered}
(-n+1)\left\{\frac{d}{d r} u(r)-\varepsilon_{r}^{p}(r)+v\left(\frac{u(r)}{r}-\varepsilon_{\theta}^{p}(r)\right)\right\} \\
+r\left\{\frac{d^{2}}{d r^{2}} u(r)-\frac{d}{d r} \varepsilon_{r}^{p}(r)+v\left(\frac{1}{r} \frac{d}{d r} u(r)\right.\right.
\end{gathered}
$$




$$
\begin{aligned}
& \left.\left.-\frac{1}{r^{2}} u(r)-\frac{d}{d r} \varepsilon_{\theta}^{p}(r)\right)\right\}-\left\{\frac{u(r)}{r}-\varepsilon_{\theta}^{p}(r)\right. \\
& \left.+v\left(\frac{d}{d r} u(r)-\varepsilon_{r}^{p}(r)\right)\right\}+\frac{\rho_{0} \omega^{2} r^{2}\left(1-v^{2}\right)}{E}=0 .
\end{aligned}
$$

Using relations in Section 2.3, the FD form of this differential equation can be expressed as follows:

$$
\begin{aligned}
(-n+1) & \left\{\frac{1}{2 r_{i}} \frac{u_{i+1}-u_{i-1}}{\Delta r}-\left(\varepsilon_{r}^{p}\right)_{i}\right. \\
& \left.+v\left(\frac{u_{i}}{r_{i}}-\left(\varepsilon_{\theta}^{p}\right)_{i}\right)\right\}+r\left\{\frac{u_{i+1}-2 u_{i}+u_{i-1}}{\Delta r^{2}}\right. \\
& -\frac{1}{2} \frac{\left(\varepsilon_{r}^{p}\right)_{i+1}-\left(\varepsilon_{r}^{p}\right)_{i-1}}{\Delta r}+v\left(\frac{1}{2 r_{i}} \frac{u_{i+1}-u_{i-1}}{\Delta r}\right. \\
& \left.\left.-\frac{u_{i}}{r_{i}^{2}}-\frac{1}{2} \frac{\left(\varepsilon_{\theta}^{p}\right)_{i+1}-\left(\varepsilon_{\theta}^{p}\right)_{i-1}}{\Delta r}\right)\right\}-\left\{\frac{u_{i}}{r_{i}}-\left(\varepsilon_{\theta}^{p}\right)_{i}\right. \\
& \left.+v\left(\frac{1}{2} \frac{u_{i+1}-u_{i-1}}{\Delta r}-\left(\varepsilon_{r}^{p}\right)_{i}\right)\right\} \\
& +\frac{\rho_{0} \omega_{i}^{2} r_{i}^{2}\left(1-v^{2}\right)}{E}=0 .
\end{aligned}
$$

Similar to the elastic solution, the FD form of the equilibrium equation for internal grid points $(i=[2, N-1])$ and the FD form of boundary conditions for $i=1, N$ are written. Finally, the system of linear algebraic equations is solved and the radial displacement of the rotating disk in the radial direction is obtained for each grid point. Now, one can achieve values of stresses and strains in the rotating disk beyond the elastic limit angular velocity. However, it should be noted that the equation cannot be solved directly, since plastic strains are path-dependent. Therefore, increments of plastic strains must be integrated or added together along the loading path to give the total plastic strains. To achieve this goal, the incremental Prandtl-Reuss stress-strain relations and the method of successive elastic solutions are used.

\subsubsection{Prandtl-Reuss equation}

In the Prandtl-Reuss equation, increments of the plastic strain are related to the value of the stress and the uniaxial stress-strain curve. Prandtl and Reuss assume that the plastic strain increment is proportional to the instantaneous stress deviation $[44,45]$ :

$$
\Delta \varepsilon_{i j}^{p}=\lambda S_{i j},
$$

where $S_{i j}$ is the deviatoric stress tensor and $\lambda$ is a nonnegative constant, which may vary throughout the loading history. These equations imply that the plastic strain increments depend on the current stress state and not on the stress increment required to reach this state. One can define effective plastic strain increment and effective stress, respectively, as:

$$
\Delta \varepsilon_{e q}^{p}=\sqrt{\frac{2}{3} \Delta \varepsilon_{i j}^{p} \Delta \varepsilon_{i j}^{p}}, \quad \sigma_{e}=\sqrt{\frac{2}{3} S_{i j} S_{i j}} .
$$

Using these definitions, $\lambda$ is determined as:

$$
\lambda=\frac{3}{2} \frac{\Delta \varepsilon_{e q}^{p}}{\sigma_{e}}
$$

and the Prandtl-Reuss relations become [43,44]:

$$
\Delta \varepsilon_{i j}^{p}=\frac{3}{2} \frac{\Delta \varepsilon_{e q}^{p}}{\sigma_{e}} S_{i j}
$$

These equations are used together with the von Mises yield criterion. Yielding begins when the effective stress reaches the yield stress determined from a uniaxial tensile test. In this study, a linear strain hardening stress-strain curve is selected for AL2024-T351.

\subsubsection{The method of successive elastic solution}

To solve the governing differential equation of the rotating disk beyond elastic limit angular velocity, an iterative process, known as the method of successive elastic solution [45], is used. The method involves breaking the loading path into a number of small increments. The rotating disk is assumed to be under the plane stress loading condition. For each new increment of loading, the solution is obtained as follows $[45,46]$ :

Step 1: For each thickness, geometric parameter, $n$, and the elastic limit angular velocity $\left(\omega_{\text {crit,e }}\right)$, at which the plastic yielding begins, are calculated from the elastic solution;

Step 2: Assuming that the final angular velocity is $\omega_{f}$, the loading path is divided into $M$ steps and each angular velocity increment is $\Delta \omega=\left(\omega_{f}-\omega_{\text {crit }, e}\right) / M$. The angular velocity at the $j$ th loading step is found to be:

$$
\omega_{j}=\omega_{\text {crit }, e}+j \Delta \omega .
$$

Step 3: Initial values of radial and tangential plastic strain increments, i.e. $\Delta \varepsilon_{r, i j}^{p}$ and $\Delta \varepsilon_{\theta, i j}^{p}$, respectively, are assumed and added to the accumulated plastic strains obtained from previous loading steps at any increment of the radius. In the initial loading step, the accumulated plastic strains are zero. The radial and tangential plastic strains are:

$$
\varepsilon_{r}^{p}=\sum_{k=0}^{j-1} \Delta \varepsilon_{r, i k}^{p}+\Delta \varepsilon_{r, i k}^{p}
$$




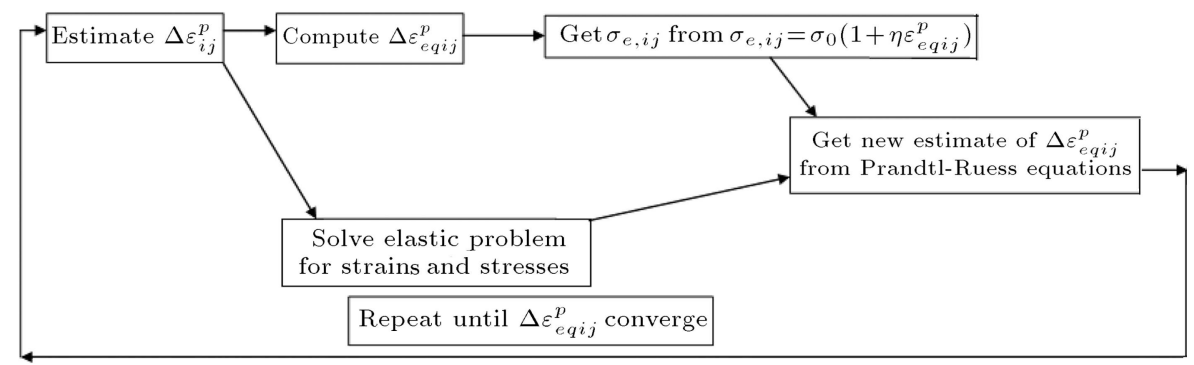

Figure 5. A schematic diagram of the iteration scheme without the damage effect.

$$
\varepsilon_{\theta}^{p}=\sum_{k=0}^{j-1} \Delta \varepsilon_{\theta, i k}^{p}+\Delta \varepsilon_{\theta, i k}^{p}
$$

Subscripts $i$ and $j$ refer to the point along the radius and the loading step, respectively. The plastic strain increment in the thickness direction is obtained from an incompressibility condition, that is:

$$
\Delta \varepsilon_{z, i j}^{p}=-\left(\Delta \varepsilon_{r, i j}^{p}+\Delta \varepsilon_{\theta, i j}^{p}\right) .
$$

Step 4: The equivalent plastic strain increment is then calculated as:

$$
\begin{aligned}
& \Delta \varepsilon_{e q, i j}^{p}=\frac{2}{\sqrt{3}}\left[\left(\Delta \varepsilon_{r, i j}^{p}-\Delta \varepsilon_{\theta, i j}^{p}\right)^{2}\right. \\
& \left.\quad+\left(\Delta \varepsilon_{\theta, i j}^{p}-\Delta \varepsilon_{z, i j}^{p}\right)^{2}+\left(\Delta \varepsilon_{r, i j}^{p}-\Delta \varepsilon_{z, i j}^{p}\right)^{2}\right]^{1 / 2}
\end{aligned}
$$

Step 5: From the stress-strain curve, the effective stress is calculated at each loading step for each increment of radius:

$$
\sigma_{e, i j}=\sigma_{0}\left(1+\eta \varepsilon_{e q, i j}^{p}\right), \quad \eta=\frac{E E_{t}}{\sigma_{0}\left(E-E_{t}\right)},
$$

in which $\varepsilon_{e q, i j}^{p}=\sum \Delta \varepsilon_{e q, i j}^{p}$.

Step 6: Based on the assumption made for values of plastic strains and the angular velocity increment, the inhomogeneous governing differential equation, i.e. Eq. (34), is solved and the stress is calculated.

Step 7: Having found the value of stresses from Step 6, and the equivalent plastic strain and stress from Steps 4 and 5, a new and improved approximation is obtained for the latest increment of the plastic strains employing Prandtl-Reuss relations:

$$
\begin{aligned}
& \Delta \varepsilon_{r, i j}^{p(\text { new })}=\frac{\Delta \varepsilon_{e q, i j}^{p}}{2 \sigma_{e, i j}}\left(2 \sigma_{r, i j}-\sigma_{\theta, i j}\right), \\
& \Delta \varepsilon_{\theta, i j}^{p(\text { new })}=\frac{\Delta \varepsilon_{e q, i j}^{p}}{2 \sigma_{e, i j}}\left(2 \sigma_{\theta, i j}-\sigma_{r, i j}\right) .
\end{aligned}
$$

Step 8: The method is iterated from Step 4 until the $j$ th loading step converges. The convergence criteria of the iteration number, $L$, in each loading step, can be expressed in terms of the value of the maximum plastic strain component as follows:

$$
\frac{\left(\varepsilon_{\max }^{p}\right)_{L+1}-\left(\varepsilon_{\max }^{p}\right)_{L}}{\left(\varepsilon_{\max }^{p}\right)_{L}} \leq 10^{-5} .
$$

Step 9: The loading step is advanced by one increment and the numerical procedure is repeated from Step $2[45,46]$. A schematic diagram of the iteration scheme without the damage effect is shown in Figure 5.

\subsection{Implementation of damage evolution equations in $S A M$}

In order to apply the $\mathrm{X}-\mathrm{W}$ damage plasticity model to the semi-analytical method, the same concept presented in Section 3.1 may be used. The effect of damage variable, $D$, in weakening function $w(D)$ by the weakening factor of $\left(1-D^{\beta}\right)$ is applied to the plastic solution. This weakening function is added to Step 5 of Section 4.2.2. Hence, the value of the effective stress at each loading step and for each increment of the radius is corrected as follows:

$$
\sigma_{e, i j}=W\left(D_{j}\right) \sigma_{0}\left(1+\eta \varepsilon_{e q, i j}^{p}\right) .
$$

For the angular velocities beyond the elastic limit, once the converged value of the first increment of the loading is reached, the damage variable for grid points in the radial direction of the disk is calculated according to Eq. (9). The new increment is then imposed, and the algorithm for the method of successive elastic solution is restarted and repeated until the fracture criterion for a given point is satisfied, i.e. the integral over the damage variable reaches unity. At this time, fracture occurs and calculation is terminated. Similar to the finite element method, the corresponding angular velocity is considered to be the critical velocity for the rotating disk. A schematic diagram of the iteration scheme with the damage effect is shown in Figure 6 .

\section{Results and discussions}

\subsection{Elastic solution}

The geometry and material properties of the disk under study in this research are shown in Table 1. A detailed analysis is presented and results of the non homogonous 


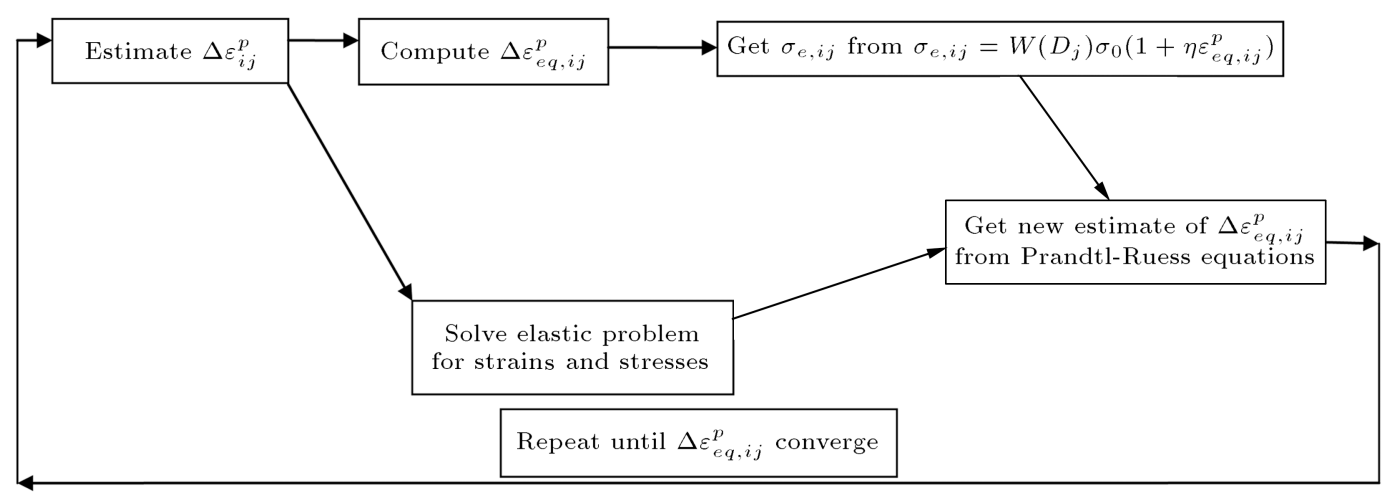

Figure 6. A schematic diagram of the iteration scheme with the damage effect.

Table 3. Convergence test for FDM with no. of grid points.

\begin{tabular}{cc}
\hline $\begin{array}{c}\text { Number of grid } \\
\text { points in FDM }\end{array}$ & $\begin{array}{c}\text { Mises stress at } \\
\boldsymbol{r}=\mathbf{0 . 1}(\mathbf{M P a})\end{array}$ \\
\hline$N=401$ & 300.06 \\
$N=301$ & 300.05 \\
$N=201$ & 300.004 \\
$N=101$ & 299.53 \\
$N=51$ & 298.86 \\
\hline
\end{tabular}

differential equation for the elastic rotating disk of variable thickness are obtained. In order to estimate the elastic limit of the angular velocity of a rotating disk at which yielding begins, one has to consider the boundary conditions. As mentioned earlier, the annular disk is mounted on a rigid shaft, hence, the boundary conditions become $u(a)=0$ and $\sigma_{r}(b)=0$.

The convergence of results obtained by the Finite Difference Method (FDM), on the basis of the elastic solution of von Mises stress at $\omega_{\text {crit }, e}=495 \mathrm{rad} / \mathrm{s}$ for an arbitrary geometric parameter, $n=0.2$, for a various number of grid points along the radius, is shown in Table 3. It is shown that the optimum number of grid points, considering the converged result and the duration of calculation, is $N=401$.

The FE simulations are carried out on the basis of axisymmetry of the loading and boundary conditions. A geometric model of the disk is made, as shown in Figure 4, and the convergence test of FE simulations is done in the same manner as that of the FDM. The convergence of results obtained from the elastic solution of von Mises stress at $\omega_{\text {crit }, e}=495 \mathrm{rad} / \mathrm{s}$ for an arbitrary geometric parameter, $n=0.2$, is shown in Table 4. As shown in this table, the optimum number of nodes, considering the converged result and the duration of calculation, is $N=7357$. The example of the mesh that is used for FE simulations of the annular rotating disk with geometric parameter $n=0.2$, is shown in Figure 7, where $h_{0}$ is the thickness at the axis of the disk. The rotating disk is thin, and the semi-analytical formulations are based on the plane
Table 4. Convergence test for FEM with no. of nodes.

\begin{tabular}{cc}
\hline $\begin{array}{c}\text { Number of nodes } \\
\text { in FE mesh }\end{array}$ & $\begin{array}{c}\text { Mises stress at } \\
\boldsymbol{r}=\mathbf{0 . 1} \text { (MPa) }\end{array}$ \\
\hline$N=7353$ & 300.015 \\
$N=6485$ & 300.01 \\
$N=4671$ & 299.9 \\
$N=2034$ & 298.02 \\
$N=234$ & 293.73 \\
$N=102$ & 289.52 \\
\hline
\end{tabular}

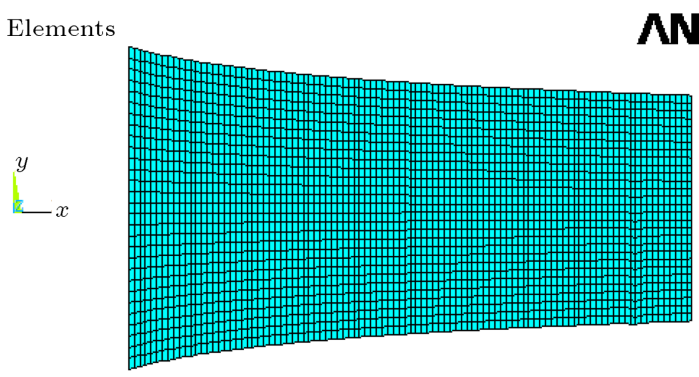

Figure 7. Axisymmetric finite element mesh of the rotating disk.

stress assumption. The thickness of the disk is assumed to be sufficiently small compared to its diameter, so that the plane stress assumption is justified. As seen in the Navier form of elastic and plastic equilibrium equations for annular disks with variable thickness in semi-analytical methods in Eqs. (31) and (36), $h_{0}$ is not included in these equations. In the finite element simulation, $h_{0}$ should be considered small enough, so that the stress in the thickness direction of the disk approaches zero and becomes negligibly small.

The elastic limit angular velocity for different values of geometric parameter, $n$, is shown in Table 5 . In this table, $\omega_{\text {crit,e }}$ is the elastic limit of the angular velocity and $r_{\left(\sigma_{e}\right)_{\max }}$ is the radius of the disk with the maximum value of the von Mises stress at $\omega_{\text {crit,e }}$. The von Mises stress at this angular velocity must be set equal to the yield stress of the disk material and that is the starting point for the yield state. The results 


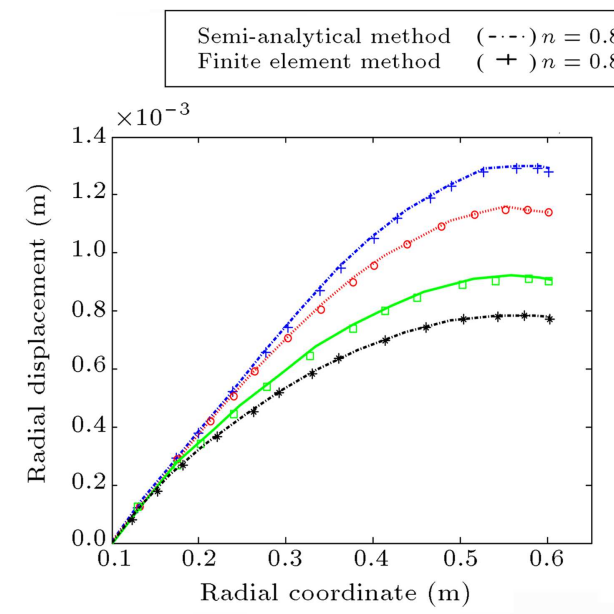

(a)

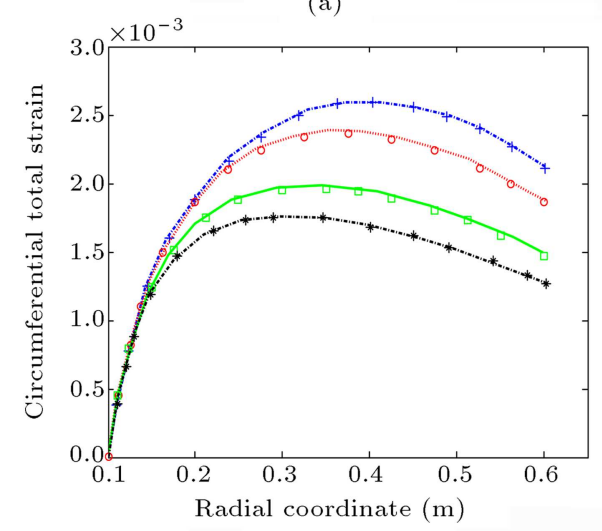

(c)

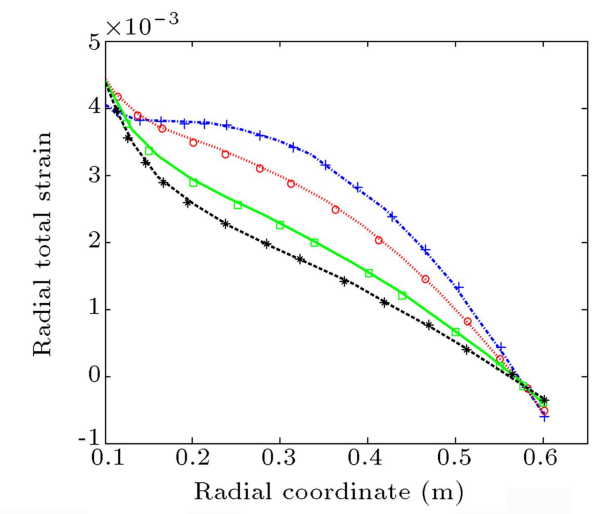

(b)

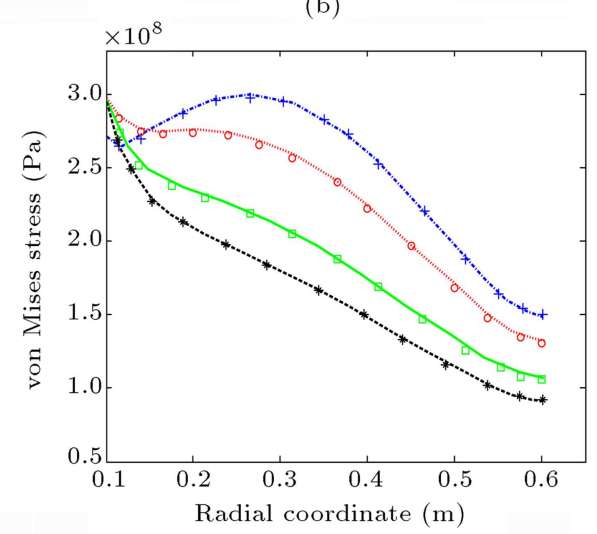

(d)

Figure 8. Comparison of elastic solutions by semi-analytical and finite element method with different $n$ at $\omega_{\text {crit,e}}$ : (a) Radial displacement; (b) radial total strain; (c) circumferential total strain; and (d) von Mises stress.

Table 5. Elastic limit angular velocity and radius of the disk with maximum von Mises stress for different values of $n$.

\begin{tabular}{ccc}
\hline $\begin{array}{c}\text { Geometric } \\
\text { parameter } \\
\boldsymbol{n}\end{array}$ & $\begin{array}{c}\boldsymbol{\omega}_{\text {crit,e }} \\
(\mathbf{r a d} / \mathbf{s})\end{array}$ & $\begin{array}{c}\text { Radius with } \\
\text { maximum von Mises } \\
\text { stress }\left(\boldsymbol{\sigma}_{\boldsymbol{e}}\right)_{\max }\end{array}$ \\
\hline$n=0$ & 440 & $r_{\left(\sigma_{e}\right)_{\max }}=0.1$ \\
$n=0.2$ & 495 & $r_{\left(\sigma_{e}\right)_{\max }}=0.1$ \\
$n=0.5$ & 585 & $r_{\left(\sigma_{e}\right)_{\max }}=0.1$ \\
$n=0.8$ & 655 & $r_{\left(\sigma_{e}\right)_{\max }}=0.27$ \\
\hline
\end{tabular}

for the radial displacement, total radial strain, total circumferential strain and von Mises stress obtained by the semi-analytical and finite element methods are shown in Figure 8. The results are obtained using the elastic limit angular velocity, $\omega_{\text {crit,e }}$, and different values of the geometric parameter, $n$.

As demonstrated in Figure 8, results obtained by the two methods are in very good agreement. It can be seen that the von Mises stress, $\sigma_{e}$, reaches its maximum value at the inner surface of the disk, i.e. at $r=a$ for $n=0,0.2,0.5$, and yielding begins from this surface. However, for $n=0.8$, the maximum von Mises stress occurs at $r=0.27$. It is observed from Table 5 that as the geometric parameter, $n$, increases, the value of the elastic limit angular velocity increases. This means that by considering variable thickness for the annular disk, the critical strain-stress level is increased and plastic deformation is delayed. The agreement observed between results obtained by these two methods shows their ability to simulate elastic deformation of a rotating disk in its general form. The results of this section are used for the plastic analysis.

\subsection{Plastic solution}

A numerical study of the plastic deformation of a rotating annular disk with variable thickness, with and without damage effect, is presented next. In this study, the plane stress condition is considered and the plastic strain in the thickness direction is not assumed to be equal to zero $\left(\varepsilon_{z}^{p} \neq 0\right)$. The failure load, i.e. the damage limit angular velocity of the rotating disk, is not directly predicted by the Ansys program, the common Prandtl-Reuss relations or the method of successive elastic solution, since the failure criterion is not included in the material properties. 
However, it would be possible to find the target speed by employing an appropriate failure model. In this paper, we use both plasticity and damage effects to estimate the damage limit angular velocity $\left(\omega_{\text {crit }, D}\right)$ of the rotating disk. According to the continuum damage mechanics, the fracture initiates in the disk when the value of the non-dimensional damage parameter, $D$, becomes equal to unity.

First, results of the semi-analytical and finite element methods of the rotating annular disk for an arbitrary geometric parameter, $n=0.2$, at different angular velocities, with and without damage effects, are obtained and compared. After validation of the two proposed methods, a parametric study for different values of geometric parameter, $n$, is carried out. The radial strain component has been adopted as the convergence criterion in the successive elastic solution, since it has the highest value among other strain components, as shown earlier in the elastic solution.

Now, results of the disk with geometric parameter $n=0.2$ rotating at the plastic limit angular velocity $\left(\omega_{\text {crit }, p}\right)$ obtained by these two methods, without damage effect, is compared. The plastic limit angular velocity is defined as the speed at which the plastic deformation is reached at the outer surface of the disk. According to the classical theory of plasticity, at this angular velocity, the plastic flow reaches the outer

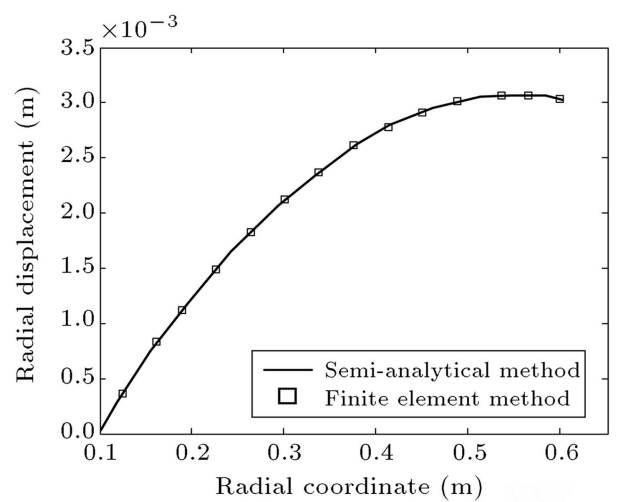

(a)

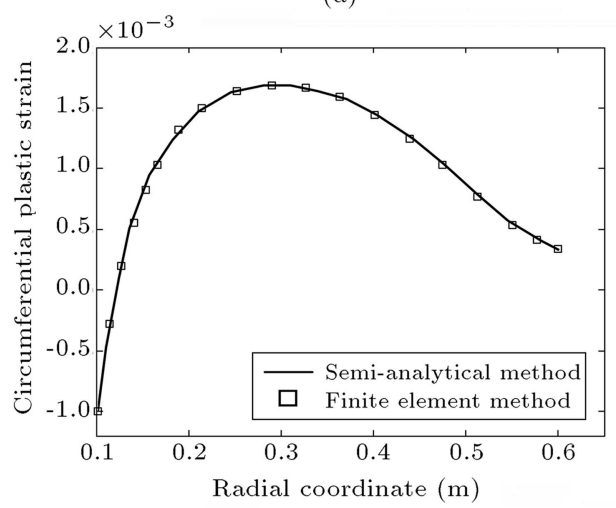

(c) surface of the disk and no elastic portion remains in the disk to withstand the load and restrict the plastic flow. Hence, at this angular velocity, plastic failure occurs and the disk fails. However, in the case of strain hardening material, and without considering the damage effect, the finite element program will continue until an angular velocity at which it becomes unstable. This angular velocity is indicated as $\omega_{\text {unstable. The }}$ values of these angular velocities for different geometric parameters are shown in Table 6 . The results obtained for the radial displacement, radial and circumferential plastic strains and the von Mises stress by semianalytical and finite element methods are compared in Figure 9. The reported results are based on the plastic limit angular velocity, $\omega_{\text {crit }, p}$, and the geometric parameter, $n=0.2$.

Table 6. Variation of $\omega_{\text {crit }, p}, \omega_{\text {crit }, D}, \omega_{\text {unstable }}$ and radius of disk with $D_{\max }$ for different geometric parameters $n$.

\begin{tabular}{ccccc}
\hline $\begin{array}{c}\text { Geometric } \\
\text { parameter } \\
\boldsymbol{n}\end{array}$ & $\begin{array}{c}\boldsymbol{\omega}_{\text {crit }, \boldsymbol{p}} \\
(\mathbf{r a d} / \mathbf{s})\end{array}$ & $\begin{array}{c}\boldsymbol{\omega}_{\text {crit }, \boldsymbol{D}} \\
(\mathbf{r a d} / \mathbf{s})\end{array}$ & $\begin{array}{c}\boldsymbol{\omega}_{\text {unstable }} \\
(\mathbf{r a d} / \mathbf{s})\end{array}$ & $\begin{array}{c}\text { Radius } \\
\text { with } \\
\boldsymbol{D}_{\max }\end{array}$ \\
\hline$n=0$ & 740 & 980 & 2100 & $r_{D_{\max }}=0.1$ \\
$n=0.2$ & 800 & 1078 & 2980 & $r_{D_{\max }}=0.13$ \\
$n=0.5$ & 830 & 1182 & 3220 & $r_{D_{\max }}=0.22$ \\
$n=0.8$ & 890 & 1260 & 2800 & $r_{D_{\max }}=0.28$ \\
\hline
\end{tabular}

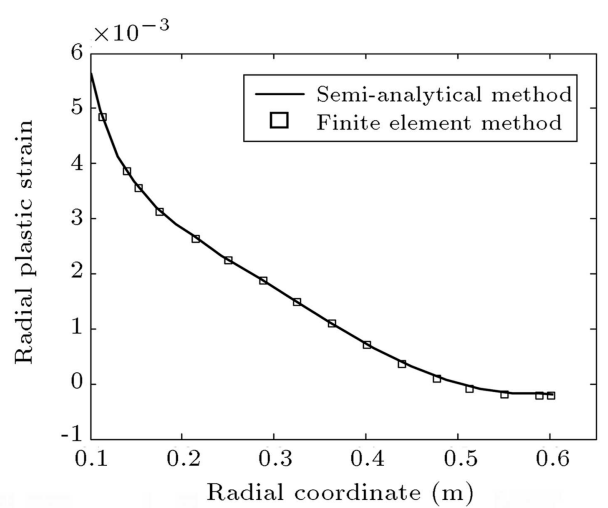

(b)

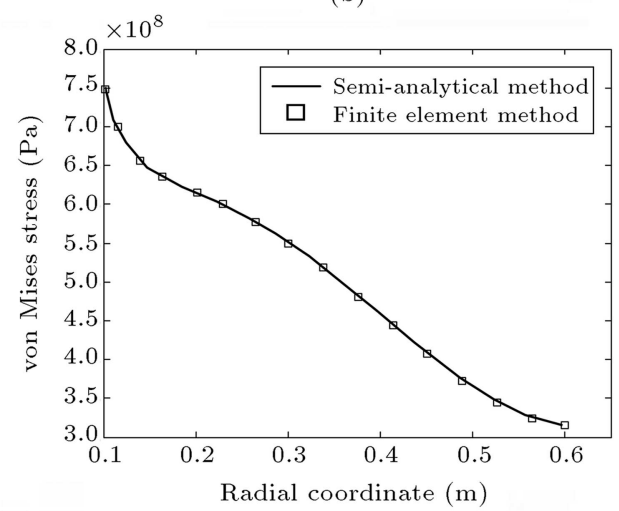

(d)

Figure 9. Comparison of plastic solution by semi-analytical and finite element method, $n=0.2$ at $\omega_{\text {crit }, p}$ without damage effect: (a) Radial displacement; (b) radial plastic strain; (c) circumferential plastic strain; and (d) von Mises stress. 


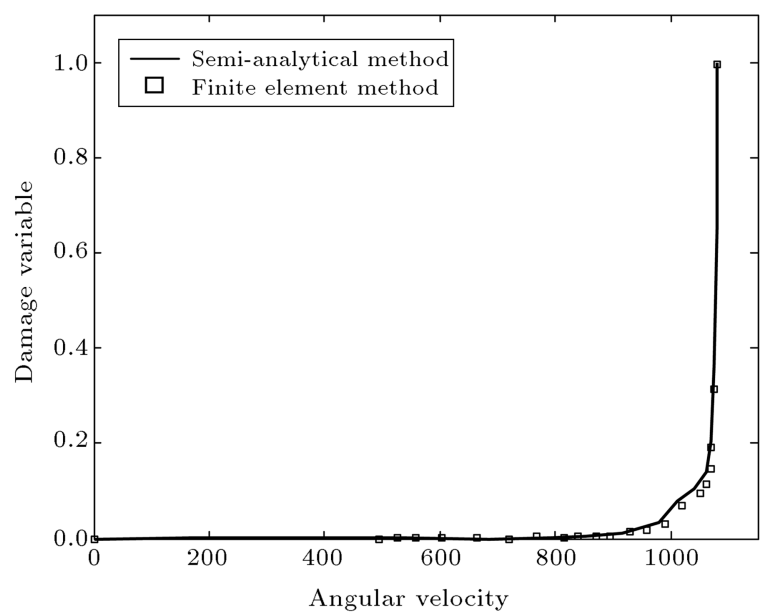

Figure 10. Variation of ductile damage parameter versus angular velocity of the rotating disk, $n=0.2$.

As shown in Figure 9, these two methods can very well describe the plastic behavior of the rotating disk without the damage effect, and results are in very good agreement. It is found that the radial strain is one of the largest plastic strain components and the maximum von Mises stress occurs at the inner surface of the disk rotating at $\omega_{\text {crit }, p}$ for $n=0.2$. In the following section, the failure analysis of the rotating disk using these two methods is presented. Variation of the ductile damage parameter versus the angular velocity of the rotating disk until fracture occurs is shown in Figure 10. The two methods are found to be in very good agreement in prediction of the damage parameter.

It is also observed from the figure that at the vicinity of $\omega_{\text {crit, } p}$, the value of the damage parameter is very small and the damage effect can be ignored. However, beyond this limit, the value of the damage parameter increases rapidly and finally reaches one. At this point, the disk cannot withstand any further loading increment and failure occurs. As shown in Figure 10, for a small value of loading increment (i.e., $\Delta \omega \approx 5 \mathrm{rad} / \mathrm{s}$ ) at the final stage, the damage parameter reaches one and, therefore, the disk ruptures. On the other hand, in the X-W damage plasticity model, the effect of applied load in the rotating disk is such that the value of $p_{\text {cut-off }}$ is approximately equal to the mean stress at the vicinity of $\omega_{\text {crit, } D}$, as shown in Figure 4. At this point, the pressure dependence function $\left(\mu_{p}(p)\right)$ from Eq. (16) falls below zero due to its logarithmic definition, and the fracture strain, $\varepsilon_{f}$, becomes zero. This means that the material cannot effectively accommodate any further plastic strain. This implies that the volumetric deformation becomes dominant and the material shatters under considerably high hydrostatic tensions. Although the material chosen for the disk is mild, the brittle type material fracture occurs in it and the disk shatters at $\omega_{\text {crit }, D}$. On an octahedral plane of cut-off pressure, the fracture locus shrinks to a single point at the triad axis.

Since the disk is made of Aluminum 2024-T351, the damage material constant is obtained experimentally, i.e. the fracture test [25,27-32]. It is observed from Table 2 that $q=1.5$ and $p_{\lim }=800 \mathrm{MPa}$, and the value of $p_{\text {cut-off }}$ for the rotating disk is found to be:

$$
p_{\text {cut-off }}=p_{\text {lim }}\left(1-e^{1 / q}\right)=-1.94 p_{\text {lim }}=-758 \mathrm{MPa} .
$$

Knowing that the mean stress, $\sigma_{m}$, is the negative value of the pressure, $p$, the damage occurs for the following range of values of the mean stress:

$$
-800 \mathrm{MPa} \leq \sigma_{m} \leq 758 \mathrm{MPa} .
$$

In the following section, semi-analytical and finite element investigations considering the damage effect are carried out. Results obtained by these methods for radial displacement, radial and circumferential plastic strain, and von Mises stress distribution are presented in Figure 11. The results are obtained on the basis of the damage limit angular velocity, $\omega_{\text {crit, } D \text {, and the }}$ geometric parameter, $n=0.2$. It is observed in these figures that the maximum effect of damage occurs in radii with the maximum value of ductile damage parameter. The mean stress level for the disk under study with different geometric parameters, i.e. $n$ at $\omega_{\text {crit, } D}$, are shown in Figure 12. According to Figure 3, the value of the mean stress at the effected radius with $D=1$ is in the vicinity of $\sigma_{m}=-p_{\text {cut-off }}=758 \mathrm{MPa}$.

Variation of the ductile damage parameter with the radius of disk at $\omega_{\text {crit }, D}$ for geometric parameter $n=0.2$, obtained by semi-analytical and finite element methods, is shown in Figure 13. It is expected from the elastic solution that the inner surface of the disk with the maximum value of von Mises stress at $\omega_{\text {crit,e }}$ will have the highest value of ductile damage parameter. However, it can be found from Figure 13 and Table 6 that the point with the highest value of damage parameter is at some position inside the disk, i.e. $r_{D_{\max }}=0.13$.

The variation of Lode angle and pressure dependence function along the radius of the disk for an arbitrary geometric parameter, $n=0.2$ at $\omega_{\text {crit }, D}$, is shown in Figure 14. According to results shown in Table 6 , the radius of the disk with highest value of damage parameter $(D=1)$ for $n=0.2$ is at $r=0.13$. As shown in Figure 14, at this radius, the value of the Lode angle parameter is minimized and the value of the pressure dependence parameter is also near to zero. Certainly, at this radius, the value of the fracture strain approaches zero. In other radii, the value of the Lode angle is high, so, the effect of the pressure dependence function is reduced.

Despite difficulties experienced in application of the algorithm of the damage plasticity model in the 


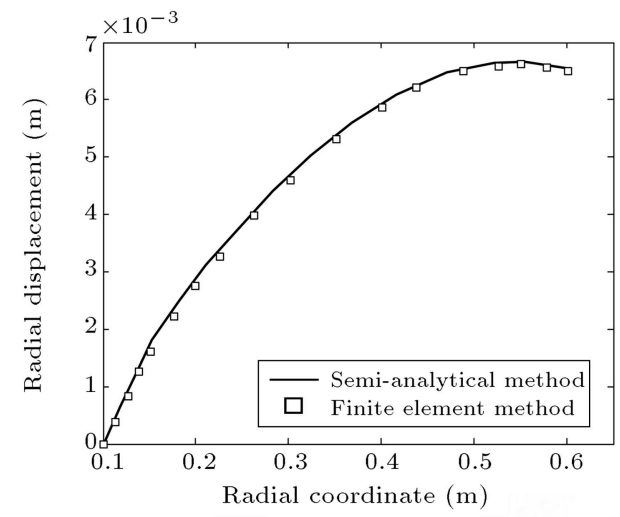

(a)

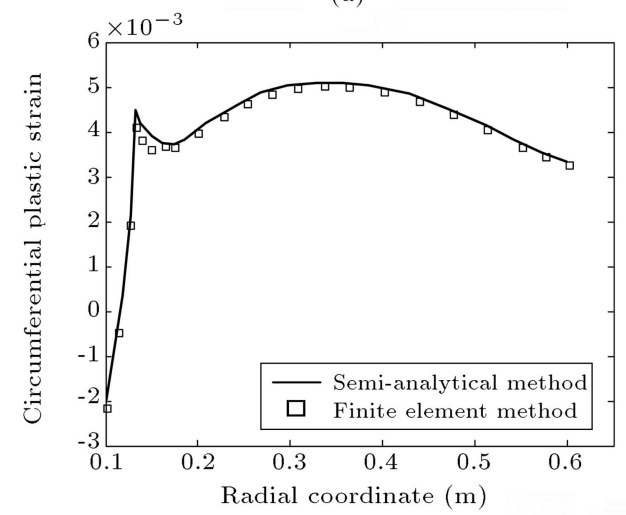

(c)

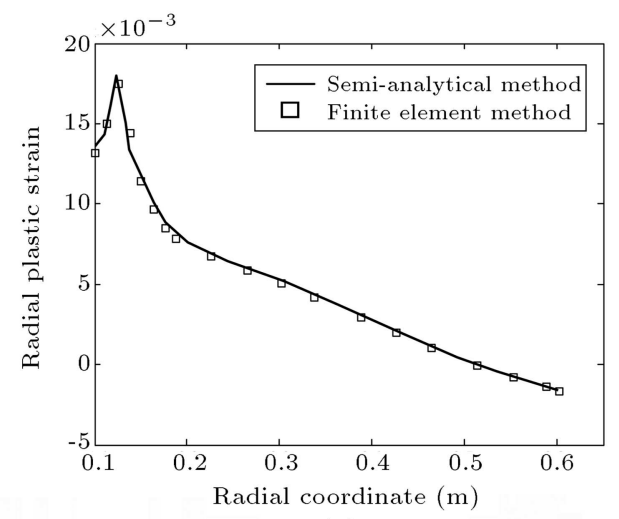

(b)

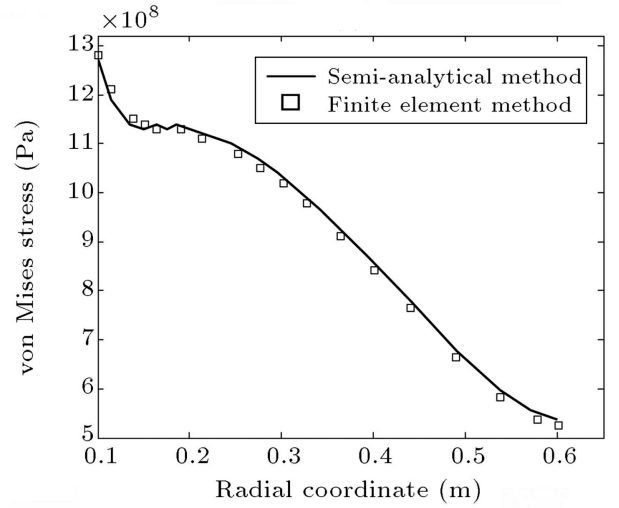

(d)

Figure 11. Comparison of results for a rotating annular disk by semi-analytical and FE methods with damage effect, $n=0.2$ at $\omega_{\text {crit }, D}:$ (a) Radial displacement; (b) radial plastic strain; (c) circumferential plastic strain; and (d) von Mises stress.

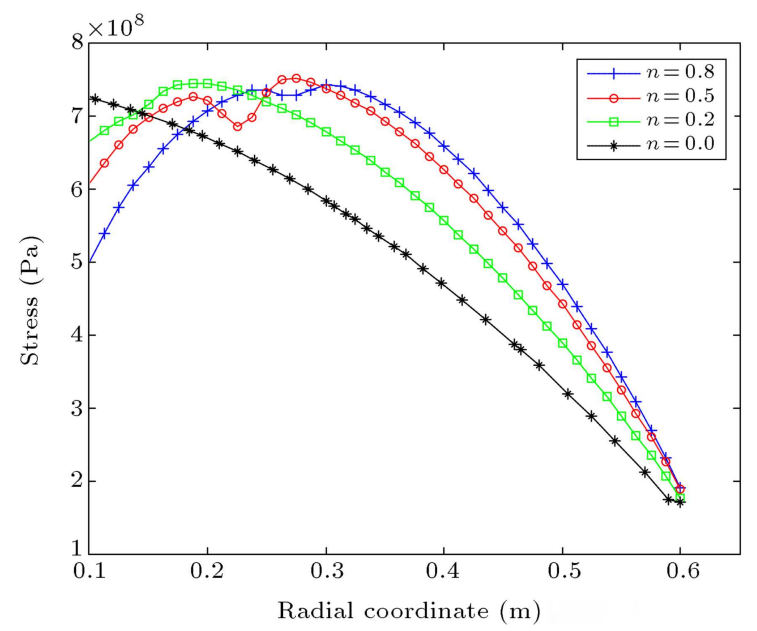

Figure 12. Variation of mean stress along the radius of the disk with damage effect for a different geometric parameter $n$ at $\omega_{\text {crit }, D}$.

semi-analytical and finite element methods, results obtained by the two methods show an excellent degree of agreement. In the semi-analytical method, the load is applied in small incremental values, requiring more computational effort and longer solution timing in comparison with the finite element method. In the next section, a parametric study is made using

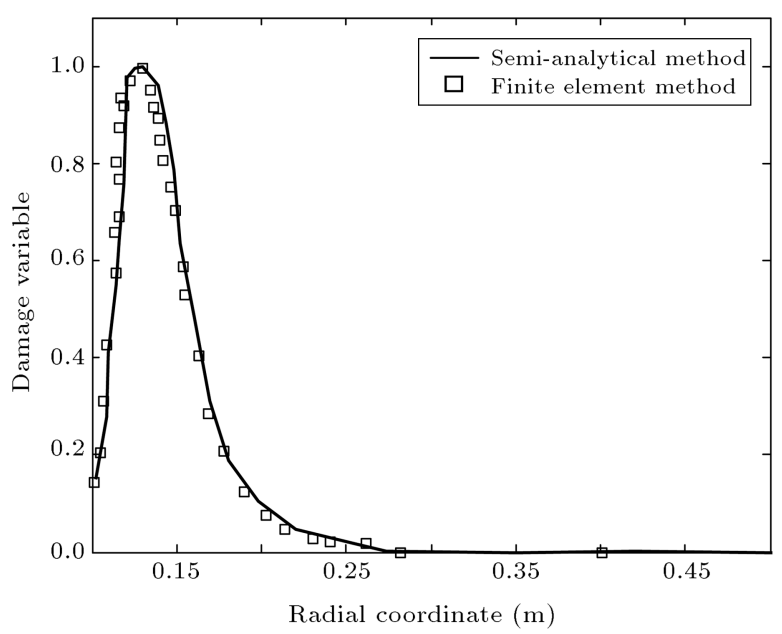

Figure 13. Variations of damage variable with radius of disk at $\omega_{\text {crit }, D}, n=0.2$.

the verified finite element method, with and without damage effect.

\subsection{Parametric study}

The main object of this section is to carry out a parametric study on the effects of the geometric parameter, $n$, with and without the damage effect, on the behavior of the rotating disk. Variation of radial 


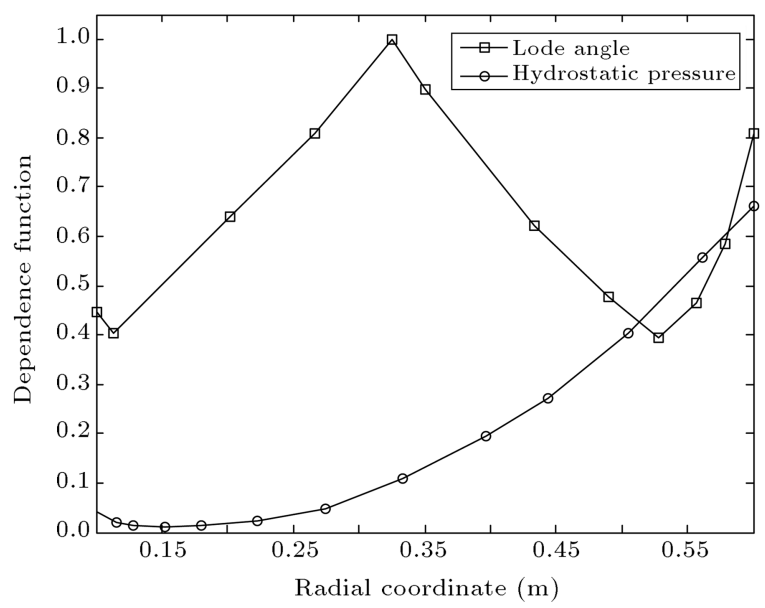

Figure 14. Variation of Lode angle-pressure dependence function along the radius of the disk for geometric parameter $n=0.2$ at $\omega_{\text {crit }, D}$.

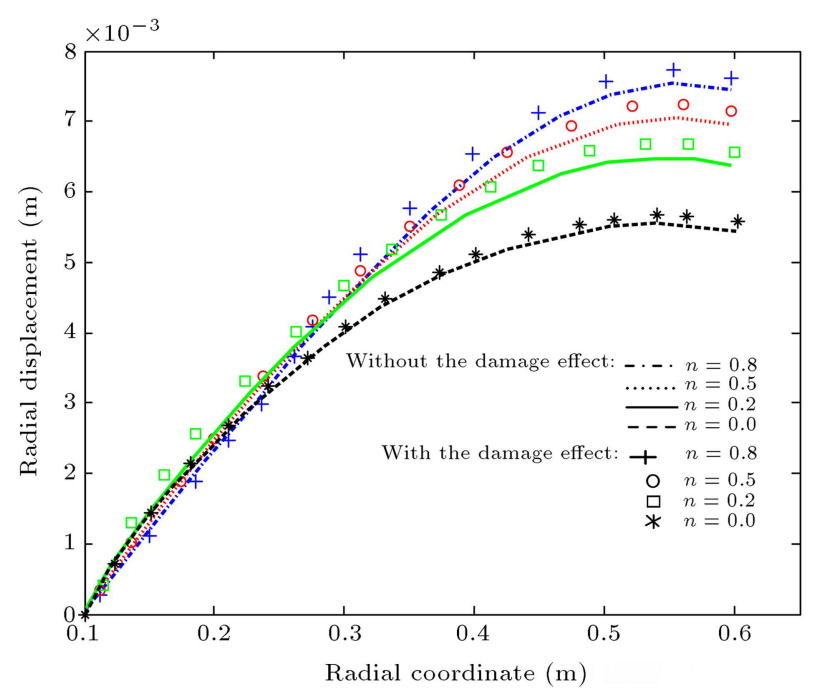

Figure 15. Variation of radial displacement of a rotating disk with $n$ at $\omega_{\text {crit }, D}$ with and without damage effect.

displacement, with geometric parameter, $n$, with and without the damage effect, is shown in Figure 15. Results obtained for the radial, circumferential and through the thickness plastic strain for different values of $n$, are shown in Figure 16. Finally, the variation of von Mises stress with the geometric parameter, with and without the damage effect, is shown in Figure 17. The reported results are based on using the damage limit angular velocity, $\omega_{\text {crit, } D}$, as indicated in Table 6 for different geometric parameters, $n$.

The radial position at which the effect of the damage parameter is the highest (i.e. $D=1$ ), for different values of the geometric parameter, $n$, is shown in Table 6. These results are verified by both semianalytical and finite element methods. It is observed from Figure 8(d) that the maximum value of von Mises stress in the elastic limit occurs at the inner radius of the disk for $n=0,0.2,0.5$. It is expected that for these geometric parameters, the highest value of the damage parameter occurs at the inner radius. For other geometric parameters, i.e. $n=0.8$, this location is shifted towards the center of the disk. When the damage effect is considered, the strength matrix for the material of the disk is corrected at each loading step by the damage variable obtained in the previous loading step with a weakening function of $W(D)=\left(1-D^{\beta}\right)$ as defined in Eq. (13). It is observed from Eq. (9) that the value of the damage variable is directly related to three parameters, namely, the accumulated plastic strain, the pressure dependence function and the Lode angle dependence function. The combined effect of these parameters causes the disk to become more nonlinear or the geometric parameter, $n$, to increase, and the position of the maximum value of the damage parameter $(D=1)$ shift towards the inner portion of the disk.

When the damage effect is considered, the von Mises stress at every affected point of the disk is decreased and, correspondingly, the value of the plastic strain is increased. It may generally be concluded that as the geometric parameter increases, the effect of ductile damage on plastic strains becomes more predominant. From Figure 17, it can be seen that in simulations with and without damage effects, as the geometric parameter $n$ increases, i.e. the rate of change of the thickness increases, the stress level in the rotating disk at the inner surface of the disk decreases. It is also found that the value of plastic strains with the damage effect at $\omega_{\text {crit, } D}$ asymptotically approaches the value of the plastic strain corresponding to $\omega_{\text {unstable }}$ of the disk without damage effect. For strain linear hardening material and without considering the damage effect, the finite element program will continue till an angular velocity at which the disk becomes unstable. This angular velocity is indicated as $\omega_{\text {unstable, which is }}$ found to be very high for working environments and an inaccurate prediction of the failure angular velocity. Hence, $\omega_{\text {unstable }}$ is not a realistic estimation of the working angular velocity of the rotating disk.

$\omega_{\text {crit, } D}$ is the damage limit angular velocity of the rotating disk that can be estimated using an appropriate failure model that considers both plasticity and damage effects. According to Table 6 , the value of this limit angular velocity is much less than $\omega_{\text {unstable. }}$ However, the maximum value of the plastic strain at this angular velocity is high and asymptotically approaches the value of the plastic strain corresponding to $\omega_{\text {unstable }}$ of the disk without damage effect. This effect is shown in Figure 16 for different geometric parameter, $n$. Therefore, it may be concluded that by considering the damage effect in the simulation, one can carry out more accurate analysis and obtain more realistic estimation of the working angular velocity of the rotating disk. 


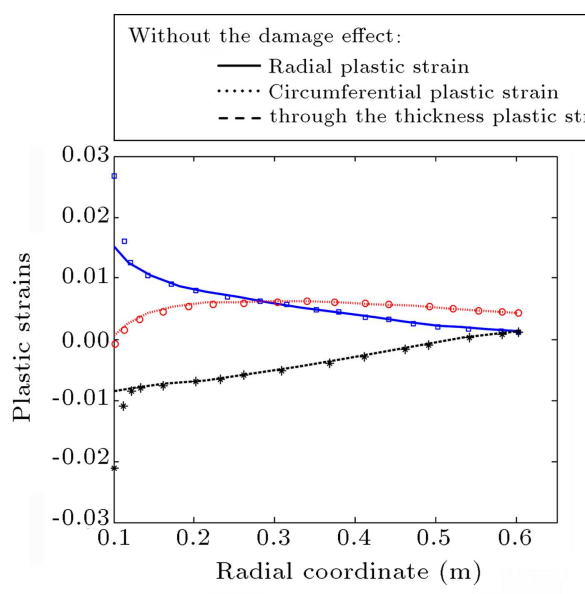

(a) $n=0$

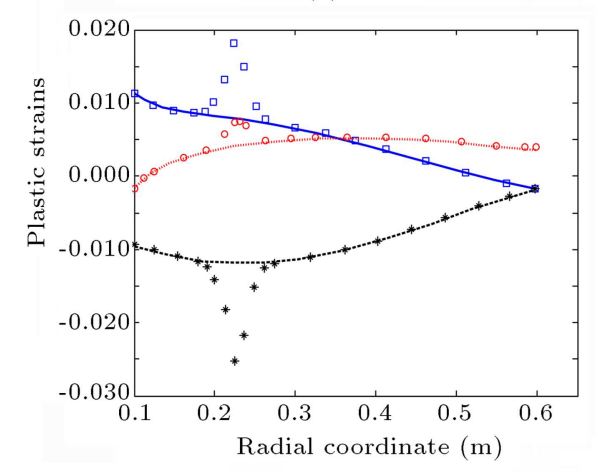

(c) $n=0.5$

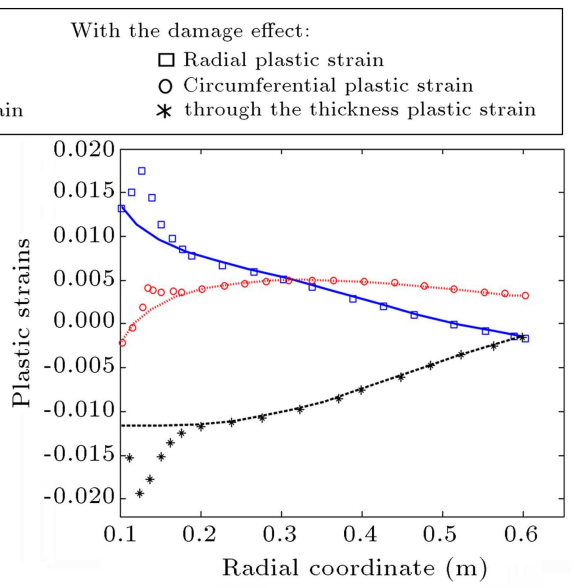

(b) $n=0.2$

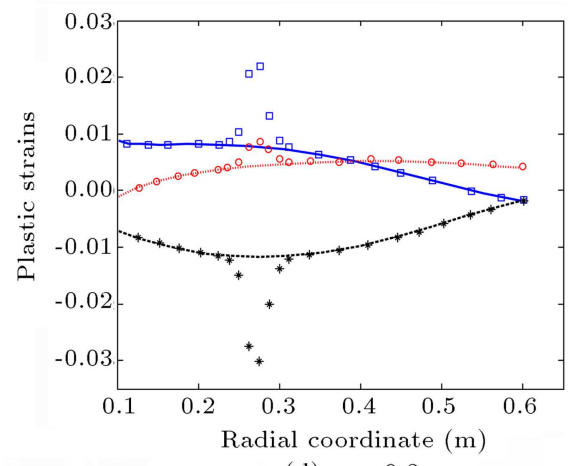

(d) $n=0.8$

Figure 16. Variations of radial, circumferential and through thickness strains of a rotating disk at $\omega_{\text {crit, } D}$ with and without damage effect: (a) $n=0$; (b) $n=0.2$; (c) $n=0.5$; and (d) $n=0.8$.

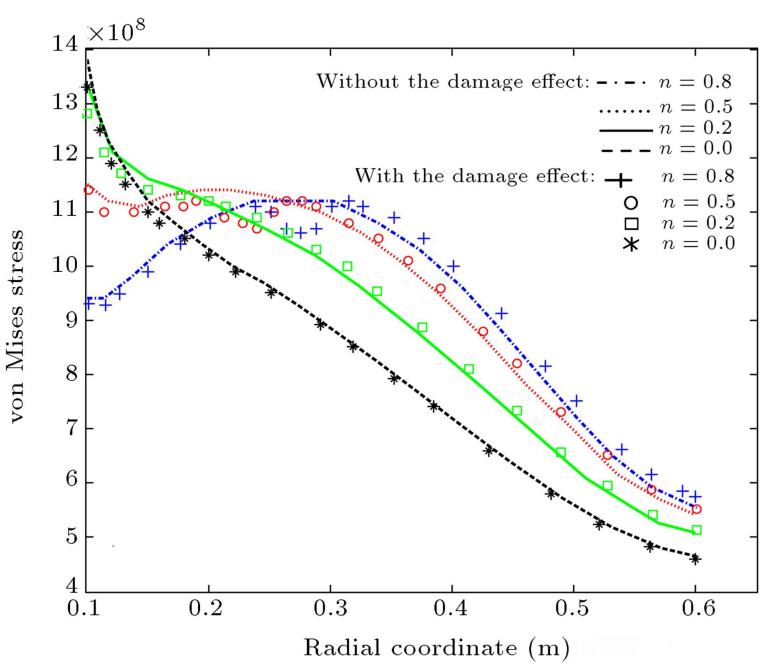

Figure 17. von Mises stress distribution of a rotating disk with $n$ at $\omega_{\text {crit, } D}$ with and without damage effect.

In Figure 18, variations of the ductile damage parameter versus the angular velocity of the rotating disk for different geometric parameter, $n$, is shown. The trend of variations of the damage parameter for different geometric parameters is almost similar. It is also observed that as the geometric parameter increases. the value of $\omega_{\text {crit }, D}$ increases.

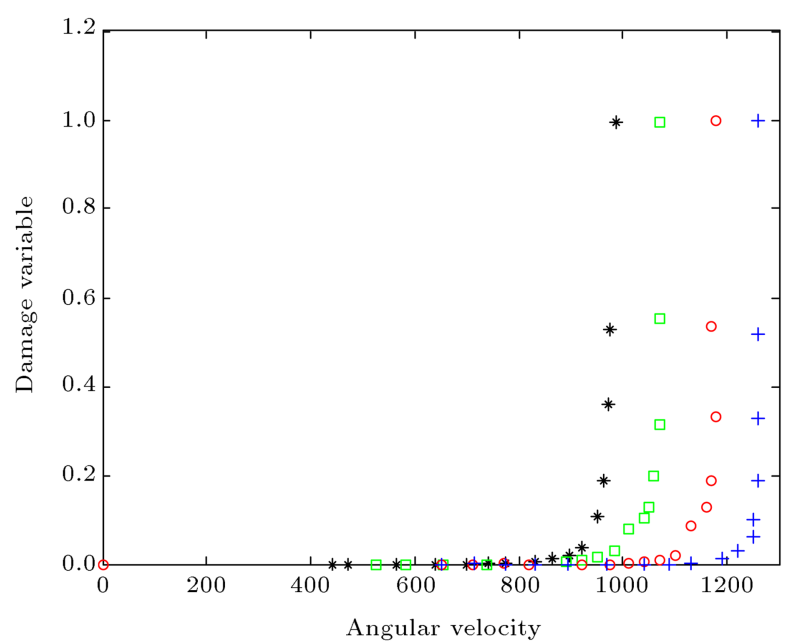

Figure 18. Variation of the ductile damage parameter versus angular velocity of a rotating disk; $n$ is a parameter.

\section{Conclusions}

Semi-analytical and finite element calculations for the elastic-plastic analysis of a rotating annular disk of variable thickness, with and without damage effects, are presented. The (X-W) damage plasticity model is introduced in both methods to obtain variations of plastic strains and the damage limit angular ve- 
locity for different values of the geometric parameter, $n$. The proposed damage plasticity model includes material deterioration, pressure sensitivity, Lode angle dependence and the nonlinear damage evolution law. The numerical implementation of the damage evolution equation is simple, with good physical understanding. The ductile damage parameter is obtained from the accumulation of plastic strains in the rotating disk and is affected by the geometric parameter and mechanical loading. The plastic strains and von Mises stress distribution are found to vary proportional to the ductile damage parameter along the radius of the disk. However, for angular velocities in the vicinity of $\omega_{\text {crit }, p}$, values of the ductile damage parameter are small and the damage effect can be ignored. It is also noted that for each geometric parameter in the vicinity of the target angular velocity, the plastic strain increases rapidly for any incremental values of the order of $\Delta \omega \approx$ $5 \mathrm{rad} / \mathrm{s}$, and the damage variable rapidly reaches one. Due to this effect, the rotating disk in the vicinity of the damage limit angular velocity shatters suddenly. According to $\mathrm{X}-\mathrm{W}$ damage plasticity model, in the vicinity of the damage angular velocity, the value of the cut-off pressure becomes approximately equal to the mean stress, and the fracture envelope approaches a point in the mean stress-plastic strain space. The radius with the maximum value of ductile damage parameter is found to be at some position inside the disk for different values of $n=0.2,0.5,0.8$. However, for $n=0$, the position of the point is at the inner surface of the disk. Moreover, it is found that the damage has a reducing effect on the von Mises stress and an increasing effect on the plastic strains in radii with the maximum value of ductile damage parameter. The results of the two methods have found to be in excellent agreement for both cases, i.e. with and without the damage effect. It can be concluded that the proposed semi-analytical and finite element methods can successfully handle the elastic-plastic problem of a rotating disk under mechanical loading, and could be extended to analyze more complicated problems of combined loading cases, such as thermo-mechanical loadings of gears, turbine rotors, flywheels and other mechanical components.

\section{References}

1. Calladine, C.R., Engineering Plasticity, Oxford: Pergamon Press (1969).

2. Timoshenko, S. and Goodier, J.N., Theory of Elasticity, 3rd Edn., New York, McGraw-Hill (1970).

3. Johnson, W. and Mellor, P.B., Engineering Plasticity, Chichester, UK, Ellis Horwood (1983).

4. Gural, U. and Fenster, S.K., Advanced Strength and Applied Elasticity, 3rd Edn., London, Prentice-Hall International (1995).
5. Laszlo, F. "Rotating disks in the region of permanent deformation", Technical Report, National Advisory Committee for Aeronautics (1948).

6. Gamer, U. "Elastic-plastic deformation of the rotating solid disk", Ingenieur-Arch., 54, pp. 345-54 (1984).

7. Guven, U. "Elastic-plastic stresses in a rotating annular disk of variable thickness and variable density", Int. J. Mech. Sci., 43, pp. 1137-53 (1992).

8. Guven, U. "On the applicability of Tresca's yield condition to the linear hardening rotating solid disk of variable thickness", ZAMM, 75, pp. 397-8 (1995).

9. Guven, U. "The fully plastic rotating disk of variable thickness", ZAMM, 74, pp. 61-5 (1994).

10. Eraslan, A.N. and Orcan, Y. "Elastic-plastic deformation of a rotating solid disk of exponentially varying thickness", Mech Mat., 34, pp. 423-32 (2002).

11. Eraslan, A.N. and Orcan, Y. "On the rotating elasticplastic solid disks of variable thickness having concave profiles", Int. J. Mech. Sci., 44, pp. 1445-66 (2002).

12. Eraslan, A.N. "Inelastic deformations of rotating variable thickness solid disks by Tresca's and von Mises criteria", Int. J. Comput. Eng. Sci., 3, pp. 89-101 (2002).

13. Eraslan, A.N. "Von Mises yield criterion and nonlinearly hardening variable thickness rotating annular disks with rigid inclusion", Mech. Res. Commun., 29, pp. 339-50 (2002).

14. Eraslan, A.N. "Elastic-plastic deformations of rotating variable thickness annular disks with free, pressurized and radially constrained boundary conditions", Int. J. Mech. Sci., 45, pp. 643-67 (2003).

15. Hojjati, M.H. and Jafari, S. "Variational iteration solution of elastic non uniform thickness and density rotating disks", Far East J. Appl. Math., 29, pp. 185200 (2007).

16. Hojjati, M.H. and Hassani, A. "Theoretical and numerical analyses of rotating discs of non-uniform thickness and density", Int. J. Pressure Vessels Piping, 85, pp. 694-700 (2008).

17. Hojjati, M.H. and Jafari, S. "Semi exact solution of elastic non uniform thickness and density rotating disks by homotopy perturbation and Adomian's decomposition methods. Part I: Elastic solution", International Journal of Pressure Vessels and Piping, 85, pp. $871-8$ (2008).

18. Hojjati, M.H. and Jafari, S. "Semi-exact solution of non-uniform thickness and density rotating disks. Part II: Elastic strain hardening solution", International Journal of Pressure Vessels and Piping, 86, pp. 307318 (2009).

19. Hassani, A., Hojjati, M.H., Mahdavi, E., Alashti, R.A. and Farrahi, G. "Thermo-mechanical analysis of rotating disks with non-uniform thickness and material properties", International Journal of Pressure Vessels and Piping, 98, pp. 95-101 (2012). 
20. EkhteraeiToussi, H. and RezaeiFarimani, M. "Elastoplastic deformation analysis of rotating disc beyond its limit speed", International Journal of Pressure Vessels and Piping, 89, pp. 170-177 (2012).

21. Jafari, S., Hojjati, M.H. and Fathi, A. "Classical and modern optimization methods in minimum weight design of elastic rotating disk with variable thickness and density", International Journal of Pressure Vessels and Piping, 92, pp. 41-47 (2012).

22. Robinson, E. "Bursting tests of steam-turbine disk wheels", Trans. ASME, 66, pp. 373-390 (1944).

23. $\mathrm{Hu}, \mathrm{S}$. "Large deformation and discontinuous yield analysis of rotating disks by Abaqus software", Applied Mechanics and Materials, 656, pp. 48-49 (2011).

24. Hu, S. "Study on the elastic-plastic interface and large deformation of axisymmetric disks under rotating status", Applied Clay Science, 79, pp. 41-48 (2013).

25. Xue, L. "Ductile fracture modeling - theory, experimental investigation and numerical verification", PHD Thesis, Massachusetts Institute of Technology (2007).

26. Lemaitre, J. "A continuous damage mechanics model for ductile fracture", Journal of Engineering Materials and Technology - Trans. of the ASME, 107, pp. 83-89 (1985).

27. Xue, L. and Wierzbicki, T. "Numerical simulation of fracture mode transition in ductile plates", International Journal of Solids and Structures, 46, pp. 14231435 (2009).

28. Xue, L. and Wierzbicki, T. "Ductile fracture characterization of aluminum alloy 2024-T351 using damage plasticity theory", International Journal of Applied Mechanics, 2, pp. 267-304 (2009).

29. Xue, L. and Wierzbicki, T. "Ductile fracture initiation and propagation modeling using damage plasticity theory", Engineering Fracture Mechanics, 75, pp. 32763293 (2008).

30. Xue, L. "Damage accumulation and fracture initiation in uncracked ductile solids subject to triaxial loading", International Journal of Solids and Structures, 44, pp. 5163-5181 (2007).

31. Xue, L. and Belytschko, T. "Fast methods for determining instabilities of elastic-plastic damage models through closed-form expressions", Int. J. Numer. Meth, Engng., 84, pp. 1490-1518 (2010).

32. Xue, L. and Huang, H. "Prediction of slant ductile fracture using damage plasticity theory", International Journal of Pressure Vessels and Piping, 86, pp. 319328 (2009).

33. Gurson, A.L. "Continuum theory of ductile rupture by void nucleation and growth: Part I. Yield criteria and flow rules for porous ductile media", Journal of Engineering Materials and Technology - Trans. of the ASME, 99, pp. 2-15 (1977).

34. Tvergaard, V. "Influence of voids on shear band instabilities under plane strain conditions", International Journal of Fracture, 17, pp. 389-407 (1981).
35. Johnson, G.R. and Cook, W.H. "Fracture characteristics of three metals subjected to various strains, strain rates, temperatures and pressures", Engineering Fracture Mechanics, 21, pp. 31-48 (1985).

36. Wilkins, M.L., Streit, R.D. and Reaugh, J.E. "Cumulative-strain-damage model of ductile fracture: Simulation and prediction of engineering fracture tests", Technical Report UCRL - 53058, Lawrence Livermore National Laboratory (1980).

37. $\mathrm{Hu}, \mathrm{S}$. and Moran, B. "Cracking analysis of fracture mechanics by the finite element method of lines", Acta Mech. Sinica, 21, pp. 495-502 (2005).

38. Sukumar, N., Moran, B., Belytschko, T.B. and Black, T. "An element-free Galerkin method for threedimensional fracture mechanics", Computational Mechanics, 20, pp. 170-175 (1997).

39. Narasimhan, R., Rosakis, A.J. and Moran, B. "A three dimensional numerical investigation of fracture initiation by ductile failure mechanisms in 4340 steel", In Application of Local Fracture/Damage Models to Engineering Fracture Problems, A.J. Rosakis and J.H. Giovanola, Eds., ASME AMD (1992).

40. Mendelson, A. and Roberts, E. "Analysis of plastic thermal stresses and strains in finite thin plate of strain-hardening material", National Aeronautics and Space Administration, Washington, D.C. (1964).

41. Nakmura, S., Applied Numerical Methods with Software, Prentice-Hall international Inc (1991).

42. Singh, A.K. and Bhadauria, B.S. "Finite difference formulae for unequal sub-intervals using Lagrange's interpolation formula", Int. Journal of Math Analysis, 3, pp. 815-827 (2009).

43. Ansys engineering analysis system: Ver. 12. ANSYS, Inc.

44. Chakrabarty, J., Theory of Plasticity, Third Edn., Elsevier (2000).

45. Mendeleson, A., Plasticity: Theory and Application, The Macmillan Company, new york (1978).

46. Loghman, A. and Wahab, M.A. "Loading and unloading of thick-walled cylindrical pressure vessels of strain-hardening material", Journal of Pressure Vessel Technology, pp. 105-116 (1994).

\section{Biographies}

Reza Akbari Alashti was born in Iran, in 1963. He received a PhD degree in Mechanical Engineering, Applied Design, from Tarbiat Modares University, Tehran, Iran, in 2006, which focused on the limit load analysis of cylindrical shells with opening under combined loading using constrained minimization techniques. He is currently faculty member in the Mechanical Engineering Department of Babol University of Technology, Iran, member of the Iranian Society of Mechanical Engineering, European Society of Mechanical Engineers, and the Iranian Association of 
Naval Architecture and Marine Engineers. His research interests include stress analysis in shells and plates, nonlinear analysis of structures, limit load and damage analysis.

Sanaz Jafari was born in Bojnourd, North Khorasan, Iran, in 1984. She received her BE and MS degrees in Mechanical Engineering from the University of Mazandaran, Babol, Iran, in 2006 and 2009, respectively. She also received a $\mathrm{PhD}$ degree in Mechanical Engineering, Applied Design, from Babol University of Technology, Babol, Iran, in 2015, which focused on the investigation of stress-strain field in dented thin pipes and estimation of damage using semi-analytical method. She is currently faculty member in the Mechanical Engineering Department of University of Bojnord, Iran, and member of the Iranian Society of Mechanical Engineering. Her current research interests include the design and analysis of various structural mechanics, elasticity, plasticity, fracture and damage mechanics, contact mechanic, structural analysis, finite element method, plates and shells, and indentation analysis, including stress-strain analysis and estimation of damage in indented pipes. 